\title{
A homogenization method for shape and topology optimization
}

\author{
Katsuyuki Suzuki and Noboru Kikuchi \\ The University of Michigan, Ann Arbor, MI 48109, USA \\ Received 26 July 1989 \\ Revised manuscript received 3 January 1991
}

\begin{abstract}
Shape and topology optimization of a linearly elastic structure is discussed using a modification of the homogenization method introduced by Bendsoe and Kikuchi together with various examples which may justify validity and strength of the present approach for plane structures.
\end{abstract}

\section{Introduction}

Structural optimization has recently received wide range attention in computer aided design. One of the reasons is rapid development of sophisticated but inexpensive engineering workstations with graphic capabilities, multi-windows and fast computation. The other reason is acceptance by people in industry of various computer aided engineering systems including reliable analysis capability such as finite element, finite difference and boundary element methods which are supported by geometric modeling capability with automatic mesh generation methods. The notion of structural optimization was widely discussed by structural engineers in the early 70s right after the rapid development of finite element methods. However, it could not provide practical means to design structures except in aeronautical engineering, because of lack of flexible geometric modeling and handy interactive graphic display capabilities at that time. Since most of structural design of machine parts involves heavily their geometric representation, in other words, their shape, structural design must deal with shape optimization, while most of aeronautical applications are based on frame structures with shell reinforcements which need only the concept of sizing optimization dealing with, for example, the sizes of the cross section of frames as well as the thickness of shells. It is clear that geometric representation of the structural configuration is not required in sizing optimization, and thus, its development may not be bounded by the capability of geometric modeling.

A modern theory of structural optimization based on mathematical programmings and sensitivity analysis was developed by Schmit [1] and Fox [2] in the early 60s, although the concept of fully stressed design was widely applied in design practice without solid mathematical justification but the engineers' intuition. Prager and Taylor [3] made justification of the fully stressed design for a class of structural optimization problems by deriving their optimality criteria, whose direct use in constructing optimization algorithms leads the so-called optimality criteria method in contrast to the mathematical programming method, using variational 
methods such as Lagrange multipliers and calculus of variations. The structural optimization in the 60s was restricted mostly to sizing problems of frame structures, despite that layout problems were also solved by Prager [4] for a very restricted class of structures as an extension of the concept of Michell trusses [5]. A layout problem may be formulated by finding the best possible layout of frames in a design domain to transmit applied forces to given supports by minimizing an objective function while the design constraints are satisfied. In general, the sizes of the cross section of frames are fixed in layout problems, while the location of joints and the length of frames are design variables. On the other hand, the sizes of the cross section become design variables in sizing problems, while the location of joints and the length of frames are fixed. There have been few computational works using finite element methods related to layout problems. Such a problem is solved by Svanverg [6] using mathematical programming and finite element methods for the limited case that the topology of a frame structure is fixed while the location of joints is the design variable. Most of the layout problems of trames have been solved analytically based on Prager's approach. Thus the range of covered area must be very restricted. To overcome this limitation, Rozvany [7] has made extensive exploration of various approaches to solve such problems.

Sizing problems for static frames can be solved straightforward at present by using sensitivity analysis and an appropriate mathematical programming method for optimization, since a strong tie to geometric modeling with automatic mesh generation is not required when finite element methods are applied to obtain displacements and stresses. For the fixed geometry, i.e., for the fixed nodal location and element connectivity, finite element analysis must be performed repeatedly only by varying the cross-sectional properties of frames to reach the optimum. Thus, research on sizing problems has been concentrated to optimization algorithms and methods to compute sensitivity, see, for example, [8-10], while Grierson [11] works with a mixed discrete problem that deals with discrete design variables as well as continuous design variables. Another direction of research is to improve sensitivity information by computing the second order sensitivity, see [12]. Sizing problems for frames in the context of eigenvalue problems, are solved by Olhoff [13] who solved the case that repeated eigenvalues are generated in design process, and then sensitivity may loose its sense. Once sizing problems are extended to plates/shells, the nature of the optimum becomes far from clear as Cheng and Olhoff [14] pointed out. Unless the gradient of the thickness distribution is restricted to be bounded, infinitely many and thin libs appear in the optimal structure which must be appropriately modeled to solve displacements and stresses using an anisotropic plate/shell model which may be obtained by applying the homogenization method.

Shape optimization problems are solved by Zienkiewicz and Campbell [15] in 1973, and extensive works have been published since then. Details of such publications are well surveyed in [16]. The difficulty of shape problems arises from the fact that the geometry of a structure is the design variable. This means that a finite element model associated to a structure must be changed in a process of optimization. If such a change is sufficiently small, the problem can be solved easily by applying sensitivity analysis and an appropriate optimization method, since such a change of the location of control points of the boundary shape or nodal points on the design boundary can be proportionally transferred to internal nodes of the finite element model so that convexity of each finite element is well preserved. However, if the design change becomes large, it becomes difficult to vary the finite element model without introducing excessively distorted elements which imply a large amount of approximation error in 
computation of stress. Generating well 'designed' finite element models by varying location of nodal points is, in general, a too demanding task if an automatic remeshing capability is not embedded in a shape optimization program. In this sense, the approach taken by Botkin and Bennett [17] is a natural choice, that is, an automatic mesh generation method is combined in the shape optimization program in order to deal with any amount of design change despite the additional cost in computation.

Most shape optimization problems are restricted in the case that topology of the design domain is maintained in an optimization process. In other words, the initial topology of a structure is equal to the final one. However, there are many cases that internal holes can be introduced to reduce the weight of a structure without violating design constraints. Automatic generation of holes in a design domain is, at this moment, impossible although automatic remeshing capabilities are embedded, because such capabilities cannot manage automatic topological changes of the domain yet. Thus, if the topology of the design domain would be changed to make internal holes, it is necessary to terminate the optimization process and then to restart after modifying the finite element model by generating holes. This means that varying the shape of the domain is insufficient for optimization involving topology. To overcome this limitation, an intuitive approach may be considered using a 'fixed' finite element model in which less stressed finite elements are assigned artificially very soft material so that holes can be approximately realized. This approach, however, possesses difficulties to be justified. For example, algorithms to identify less stressed elements which are 'removed' from the design are not uniquely defined and their convergence may strongly depend on the finite element models applied. That is, there is too much ambiguity in this intuitive approach. To sophisticate this naive idea, Bendsøe and Kikuchi [18] introduced a homogenization method that utilizes infinitely many microscale holes in a design domain rather than removing the whole of a finite element based on the Cheng and Olhoff's approach in sizing problems for plates/shells. Recent work by Bendsøe [19] also uses this technique. Also Rozvany [20] and coworkers' $[20,21]$ work of layout optimization by continuum-based optimality criteria methods is noteworthy.

In the present work, we shall examine the previous approach taken in [18j and we shall extend this based on the works by Pedersen [22] and Gibiansky and Chercaev [23] where classes of problems of optimal microstructures for 'fiber reinforced' composite materials were solved. Various example problems are solved to justify the present approach, while convergence and mesh dependence of finite element discretization are carefully examined using a certain type of example problems. By solving this variety of problems, it is shown that the present method can not only solve shape optimization problems but also topology optimization problems for linearly elastic structures using a fixed finite element model. In other words, layout problems in a generalized sense can be solved computationally for any type of linearly elastic structures.

\section{Generalized layout problem by a homogenization method}

As mentioned in the introduction, the main idea of solving a class of shape optimization problems involving varying topology, which is called a generalized layout problem in the present paper, is that infinitely many microscaie voids (holes) are introduced to form a 
possibly porous medium that yields a linearly elastic structure in some sense. An optimization problem for the generalized layout problem is defined by solving the optimal porosity of the medium identified with a design domain. If a portion of porous medium consists of only voids, structure is not placed over there. On the other hand, if no porosity is realized at another portion, a 'solid' structure must be placed over there. If porosity is not the limit values, porous medium is generated. In this sense, 'solid' material consisting of a structure is optimally distributed in a specified region so that a certain objective function is minimized under a set of constraints. Mathematically, such an optimal distribution problem of microscale voids (or 'solid' materiai) can be defined as

Minimize the objective function such as the mean compliance subject to equilibrium equations, void volume, stress/displacement constraints,

or

Minimize the objective function such as the volume of voids subject to

equilibrium equations, mean compliance, stress/displacement constraints.

In this paper, we shall consider the simplest case of the optimization problem since the main purpose here is to examine whether the new approach introduced can provide meaningful solutions to the generalized layout problem. If the optimal shape as well as the optimal topology of a structure can be identified by the present method, sophistication of optimization problems should be considered as the next step.

Suppose that the volume of microscale voids is specified in a given design domain $\Omega$, that is, the volume $\Omega_{\mathrm{s}}$ of 'solid' material distributed in the design domain is specified. For simplicity, the design domain is plane so that plane stress analysis is sufficient to compute displacements and stresses, while the shape of microscale voids is assumed to be rectangular as shown in Fig. 1.

Rectangular holes are chosen because they can realize the complete void $(a=b=1)$ and

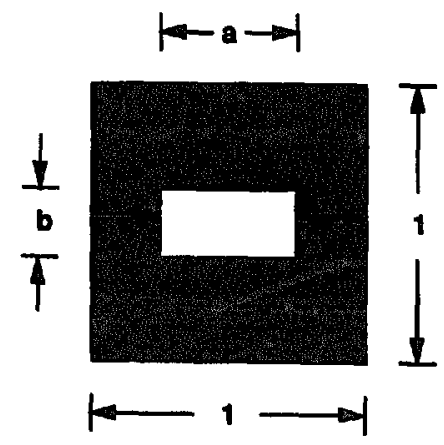

Fig. 1. A unit cell describing the microstructure with a rectangular hole. 
solid $(a=b=0)$ as well as generalized porous medium $(0<a<1,0<b<1)$. If circular holes are assumed, they cannot reach to the complete void, and then they are not appropriate to our purpose. It is noted that there are other choices to represent microscale voids such as using a generalized ellipse defined by $\left(y_{1} / a\right)^{n}+\left(y_{2} / b\right)^{n}=1$, where $a$ and $b$ are the principal radii of the ellipse and $n$ is the power defining the shape. However, in order to develop the complete void in the unit cell, both $a$ and $b$ must be 1 as well as $n$ goes to infinity for the generalized ellipse, while $a$ and $b$ need be 1 if the rectangular hole is applied. Also note that there is a possibility of using the first order microstructure in one direction and the second order microstructure in the other direction, see [24]. It can be shown that in case of low density, first/second order microstructure gives better microstructure. However, this type of microstructure is rather hard to derive material constants either analytically or numerically when Poisson's ratio is not zero. On the other hand, the first/first order microstructure numerical solution is possible for any Poisson's ratio.

Since holes are rectangular in the unit cell that characterize the microstructure of a generalized porous medium for the layout problem, their orientation is important in the macroscopic problem for stress analysis. Indeed the anisotropic elasticity tensor in the macroscopic problem strongly depends on the orientation of microscale holes. Thus, the sizes $a$ and $b$ and the orientation $\theta$ of the microscale rectangular holes are the design variables of the generalized layout problem.

Suppose that $a, b$ and $\theta$ are functions of the position $x$ of an arbitrary point of a macroscale domain of a linearly elastic 'porous' structure $\Omega$ in the two-dimensional Euclidean space $\mathbb{R}^{2}$ : $a=a(x), b=b(x)$ and $\theta=\theta(x)$. Functions $a, b$ and $\theta$ may not be so smooth, i.e., it could be true that $a \in L^{\infty}(\Omega), b \in L^{x}(\Omega)$ and $\theta$ maintains smoothness equivalent to the angle $\theta_{\mathrm{p}}$ of the principal coordinates of the stress tensor $\sigma(x)$, i.e., $\theta$ may be continuous in $\Omega$ except at a finite number of points. However, we shall assume that they are sufficiently smooth, for example, $a, b, \theta \in H^{1}(\Omega)$. Assuming that a periodic microstructure characterized by $a(x), b(x)$ and $\theta(x)$ exists in a small neighborhood of an arbitrary point $x$ in $\Omega$, and assuming that such a microstructure at $x$ need not be the same with the one at a different point $x^{*}$, see Fig. 2 which shows a schematic setting of varying microstructures, a homogenized elasticity tensor $\mathbb{E}^{\mathrm{H}}(\boldsymbol{x})$ is computed in order to solve a macroscopic stress analysis problem of a 'porous' structure.

The homogenized elasticity tensor is computed by solving the problem defined in the unit cell in which a rectangular hole is placed:

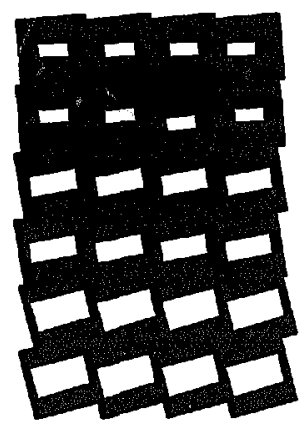

Fig. 2. Assumption of 'continuous' change of microstructures. 
Find the characteristic deformations $\chi^{(k l)}=\left\{\chi_{1}^{(k l)}, \chi_{2}^{(k l)}\right\} \in V_{Y}$ satisfying

$$
\sum_{i, j, m, n=1}^{2} \int_{Y} E_{i j m n} \frac{\partial \chi_{m}^{(k)}}{\partial y_{n}} \frac{\partial \nu_{i}}{\partial y_{i}} \mathrm{~d} Y=\sum_{i, j=1}^{2} \int_{Y} E_{i j k l} \frac{\partial \nu_{i}}{\partial y_{j}} \mathrm{~d} Y \quad \forall \nu \in V_{Y},
$$

where

$V_{Y}=\left\{\boldsymbol{v} \in \boldsymbol{H}^{1}(Y) \mid \boldsymbol{v}\right.$ is $Y$-periodic in the unit cell $\left.Y\right\}, k, l=1,2$.

Here $H_{1}(Y)$ is the Sobolev space in which an arbitrary element $v$ is square integrable in the unit cell $Y=\left(-\frac{1}{2}, \frac{1}{2}\right) \times\left(-\frac{1}{2}, \frac{1}{2}\right)$ in the $y=\left\{y_{1}, y_{2}\right\}$ coordinate system as well as its generalized first derivatives. The elasticity tensor $\mathbb{E}$ is chosen either for the plane stress or for the plane strain problem depending on the macroscopic 'porous' structure to be designed. The elasticity tensor $\mathbb{E}$ is zero if $y$ is located in the hole, and coincides with the one of the 'solid' material that is utilized to form a structure if $y$ is outside of the hole. It is noted that Young's modulus does not affect the generalized layout problem while Poisson's ratio may imply change to the optimal layout. After obtaining the characteristic deformations $\boldsymbol{\chi}^{(k l)}$, the homogenized elasticity tensor $\mathbb{E}^{\mathrm{H}}$ is computed by

$$
E_{i j k l}^{\mathrm{H}}=\sum_{m, n=1}^{2} \int_{Y}\left(E_{i j k l}-E_{i j m n} \partial \chi_{m}^{(k l)} / \partial y_{n}\right) \mathrm{d} Y .
$$

Since the sizes $\{a, b\}$ of rectangular holes are functions of the position $\boldsymbol{x}$, the homogenized elasticity tensor $\mathbb{E}^{\mathrm{H}}$ varies in $\Omega$. This means that the characteristic deformations must be obtained everywhere in the design domain $\Omega$. Solving the unit cell problem (1) everywhere is unrealistic. Thus, we shall solve (1) for several sampling points $\left\{a_{i}, b_{j} \mid i, j=1, \ldots, n\right\}$ of the sizes $\{a, b\}$ of rectangular holes, where $0 \leqslant a_{i} \leqslant 1$ and $0 \leqslant b_{j} \leqslant 1$, and we shall form a function $\mathbb{E}^{\mathrm{H}}=\mathbb{E}^{\mathrm{H}}(a, b)$ by an appropriate interpolation. The last step for the elasticity tensor for stress analysis of the macroscopic 'porous' structure is rotation of $\mathbb{E}^{\mathrm{H}}$ by the angle $\theta$. Defining the rotation matrix $\boldsymbol{R}$ by

$$
R(\theta)=\left[\begin{array}{cc}
\cos \theta & -\sin \theta \\
\sin \theta & \cos \theta
\end{array}\right],
$$

the elasticity tensor $\mathbb{E}^{\mathbf{G}}$ for stress analysis is computed by

$$
E_{i j k l}^{\mathrm{G}}(x)=\sum_{I, J, K, L=1}^{2} E_{I J K L}^{\mathrm{H}}(a(x), b(x)) R_{i l}(\theta(x)) R_{j J}(\theta(x)) R_{k K}(\theta(x)) R_{I L}(\theta(x))
$$

for $i, j, k, l=1,2$, at an arbitrary point $\boldsymbol{x}$ in $\Omega$. It is clear the $\mathbb{E}^{\mathbf{G}}$ is a function of the sizes $\{a, b\}$ and the rotation $\theta$ of microscale rectangular holes.

Stress analysis of the 'porous' structure is defined as follows:

Solve the displacement $u=\left\{u_{1}, u_{2}\right\} \in V_{\mathrm{D}}$ satisfying

$$
\sum_{i, j, k, l=1}^{2} \int_{\Omega} E_{i j k l}^{\mathrm{G}}(x) \frac{\partial u_{k}}{\partial x_{l}} \frac{\partial \nu_{i}}{\partial x_{j}} \mathrm{~d} \Omega=\sum_{i=1}^{2} \int_{\Omega} f_{i} \nu_{i} \mathrm{~d} \Omega+\sum_{i=1}^{2} \int_{\Gamma_{T}} t_{i} \nu_{i} \mathrm{~d} \Gamma \quad \forall \nu \in V_{0} .
$$


Here $V_{\mathrm{D}}=\left\{\boldsymbol{v} \in \boldsymbol{H}^{\prime}(\Omega) \mid v_{i}=g_{i}\right.$ on $\left.\Gamma_{\mathrm{D}}, i=1,2\right\}, V_{0}=\left\{v \in \boldsymbol{H}^{1}(\Omega) \mid v_{1}=0\right.$ on $\left.\Gamma_{\mathrm{D}}, i=1,2\right\}, f$ is the applied body force in $\Omega, t$ is the applied traction on a portion of the boundary $\Gamma_{\mathrm{T}}$ and $g$ is the specified displacement at the support of the body $\Gamma_{\mathrm{D}}$ which is the compliment of $\Gamma_{\mathrm{T}}$. It is noted that (4) is the expression of the principal of virtual displacement of the 'porous' structure which is a weak form of equilibrium equations and traction boundary conditions on $\Gamma_{\mathrm{T}}$. The inertia term is neglected in (4) for simplicity. $V_{\mathrm{D}}$ is a closed subset of the Sobolev space $H^{1}(\Omega)$ of square integrable functions on the domain $\Omega$, the generalized first derivatives of which are also square integrable. The specified displacement $g$ and the applied traction $t$ are sufficiently smooth functions defined on the boundary $\Gamma$ of the domain $\Omega$, say, they belong to, for example, $H^{3 / 2}\left(\Gamma_{\mathrm{D}}\right)$ and $H^{1 / 2}\left(\Gamma_{\mathrm{T}}\right)$, respectively. If the homogenized elasticity tensor $\mathbb{E}^{\mathrm{G}}$ after rotation is smooth enough, for example, if it is in $\mathbb{H}^{1}(\Omega)$, then the displacement field $u$ satisfying the principle of virtual displacement (4) can belong to $H^{2}(\Omega)$ if the boundary $\Gamma$ is sufficiently smooth. If $\mathbb{E}^{\mathrm{G}}$ is in $\mathbb{L}^{x}(\Omega)$, $u$ may belong to $\boldsymbol{H}^{3 / 2}(\Omega)$. If the solution $u$ of $(4)$ is smoother than functions in $H^{1}(\Omega)$, regular finite element approximations of (4) possess convergence properties, i.e., regular finite element approximations $u_{h}$, which are the solutions of a finite element approximation of (4), converge to the solution $u$ of the original problem (4), see $[25,26]$.

Now, let us define an optimization problem for the generalized layout that involves shape and topology of a structure. Since the purpose of the present paper is the examination of the homogenization method, we shall define the problem using the simplest objective function, the mean compliance of the structure, without any other side constraints:

$$
\underset{\substack{a, b \text { and } \theta \\ \text { subject to (4) } \\ \text { and } \\ \int_{\Omega}(1-a b) \mathrm{d} \Omega \leqslant \Omega_{\mathrm{s}}}}{\operatorname{Minimiz}} \sum_{i=1}^{2} \int_{\Omega} f_{i} u_{i} \mathrm{~d} \Omega+\sum_{i=1}^{2} \int_{\Gamma_{\mathrm{T}}} t_{i} u_{i} \mathrm{~d} \Gamma .
$$

Here $u$ is the solution of the principle of virtual displacement (4) and $\Omega_{\mathrm{s}}$ is the total volume of 'solid' material forming the 'porous' structure. In general, $\Omega_{\mathrm{s}}$ is smaller than $\boldsymbol{\Omega}$ that is the domain of the structure containing the design domain $\Omega_{\mathrm{d}}$, i.e., $\boldsymbol{\Omega}_{\mathrm{s}}<\boldsymbol{\Omega}_{\mathrm{d}}$.

If the essential boundary condition $u=g$ on $\Gamma_{\mathrm{D}}$ in (4) is resolved by applying the exterior penalty method, the solution $u$ of (4) is approximately obtained by solving the penalty formulation of (4):

$$
\begin{aligned}
& \text { Find } u_{\lambda}=\left\{u_{\lambda 1}, u_{\lambda 2}\right\} \in V \text { satisfying } \\
& \begin{array}{l}
\sum_{i, j, k, l=1}^{2} \int_{\Omega} E_{i j k l}^{\mathrm{G}}(x) \frac{\partial u_{\Lambda k}}{\partial x_{l}} \frac{\partial \nu_{i}}{\partial x_{j}} \mathrm{~d} \Omega+\sum_{i=1}^{2} \int_{\Gamma_{\mathrm{D}}} \lambda\left(u_{\lambda i}-g_{i}\right) \nu_{i} \mathrm{~d} \Gamma \\
\quad=\sum_{i=1}^{2} \int_{\Omega} f_{i} \nu_{i} \mathrm{~d} \Omega+\sum_{i=1}^{2} \int_{\Gamma_{\mathrm{T}}} t_{i} \nu_{i} \mathrm{~d} \Gamma \quad \forall \nu \in V .
\end{array}
\end{aligned}
$$

Here $V=H^{1}(\Omega)$ and $\lambda$ is a 'sufficiently' large penalty parameter. It is noted that $\boldsymbol{u}_{\lambda}$ converges to $u$ as $\lambda$ goes to infinity. If the optimization problem (5) is defined by using this penalty approximation $u_{\lambda},(5)$ can be written by 
$\underset{a, b \text { and } \theta}{\operatorname{Maximize}} \underset{\nu \in V}{\operatorname{Minimize}} \Pi_{\lambda}(\nu)$,

where

$\int_{\Omega}(1-a b) \mathrm{d} \Omega \leqslant \Omega_{\mathrm{s}}$

$$
\begin{aligned}
\Pi_{\lambda}(\nu)= & \frac{1}{2} \sum_{i, j, k, l=1}^{2} \int_{\Omega} E_{i j k l}^{\mathrm{G}} \frac{\partial \nu_{k}}{\partial x_{i}} \frac{\partial \nu_{i}}{\partial x_{j}} \mathrm{~d} \Omega+\frac{1}{2} \sum_{i=1}^{2} \int_{\Gamma_{\mathrm{D}}} \lambda\left(\nu_{i}-g_{i}\right)^{2} \mathrm{~d} \Gamma \\
& -\sum_{i=1}^{2} \int_{\Omega} f_{i} \nu_{i} \mathrm{~d} \Omega-\sum_{i=1}^{2} \int_{\Gamma_{\mathrm{T}}} t_{i} \nu_{i} \mathrm{~d} \Gamma
\end{aligned}
$$

is the total potential energy which is penalized to satisfy the essential boundary condition $\boldsymbol{u}=\boldsymbol{g}$ on the boundary $\Gamma_{\mathrm{D}}$. It is noted that the design variables $a$ and $b$ are restricted by

$$
0 \leqslant a(x) \leqslant 1 \text { and } 0 \leqslant b(x) \leqslant 1 \quad \text { in } x \in \Omega_{\mathrm{d}} .
$$

\section{Condition of the optimality criteria}

Defining a Lagrangian

$$
L(u, a, b, 6)=\Pi_{\lambda}(u)-\Lambda\left(\int_{\Omega_{\mathrm{u}}}(1-a b) \mathrm{d} \Omega-\Omega_{\mathrm{s}}\right)
$$

to the volume constraint and its Lagrange multiplier $\Lambda$ such that $\Lambda \leqslant 0$, the condition of optimality criteria can be obtained by taking formal variation of the Lagrangian with respect to the state variable $u$ and the design variables $\{a, b, \theta\}$ which are still restricted by (9):

$$
\begin{aligned}
\delta L= & \sum_{t, j, k, l=1}^{2} \int_{\Omega} E_{i j k l}^{\mathrm{G}}(x) \frac{\partial u_{k}}{\partial x_{l}} \frac{\partial}{\partial x_{j}}\left(\delta u_{i}+\frac{\partial u_{i}}{\partial a} \delta a+\frac{\partial u_{i}}{\partial b} \delta b+\frac{\partial u_{i}}{\partial \theta} \delta \theta\right) \mathrm{d} \Omega \\
& +\sum_{i=1}^{2} \int_{\Gamma_{\mathrm{D}}} \lambda\left(u_{i}-g_{i}\right)\left(\delta u_{i}+\frac{\partial u_{i}}{\partial a} \delta a+\frac{\partial u_{i}}{\partial b} \delta b+\frac{\partial u_{i}}{\partial \theta} \delta \theta\right) \mathrm{d} \Gamma \\
& -\sum_{i=1}^{2} \int_{\Omega} f_{i}\left(\delta u_{i}+\frac{\partial u_{i}}{\partial a} \delta a+\frac{\partial u_{i}}{\partial b} \delta b+\frac{\partial u_{i}}{\partial \theta} \delta \theta\right) \mathrm{d} \Omega \\
& -\sum_{i=1}^{2} \int_{\Gamma_{\mathrm{T}}} t_{i}\left(\delta u_{i}+\frac{\partial u_{i}}{\partial a} \delta a+\frac{\partial u_{i}}{\partial b} \delta b+\frac{\partial u_{i}}{\partial \theta} \delta \theta\right) \mathrm{d} \Gamma \\
& +\int_{\Omega}\left\{\sum_{i, j, k, l=1}^{2} \frac{1}{2} \frac{\partial E_{i j k l}^{\mathrm{G}}}{\partial a} \frac{\partial u_{k}}{\partial x_{l}} \frac{\partial u_{i}}{\partial x_{i}}+\Lambda b\right\} \delta a \mathrm{~d} \Omega \\
& +\int_{\Omega}\left\{\sum_{i, j, k, l=1}^{2} \frac{1}{2} \frac{\partial E_{i j k l}^{\mathrm{G}}}{\partial b} \frac{\partial u_{k}}{\partial x_{l}} \frac{\partial u_{i}}{\partial x_{j}}+\Lambda a\right\} \delta b \mathrm{~d} \Omega \\
& +\int_{\Omega}\left\{\sum_{i, j, k, l=1}^{2} \frac{1}{2} \frac{\partial E_{i j k l}^{\mathrm{G}}}{\partial \theta} \frac{\partial u_{k}}{\partial x_{l}} \frac{\partial u_{i}}{\partial x_{j}}\right\} \delta \theta \mathrm{d} \Omega+\left\{\int_{\Omega_{\mathrm{d}}}(1-a b) \mathrm{d} \Omega-\Omega_{\mathrm{s}}\right\} \delta \Lambda,
\end{aligned}
$$


where $\delta u$ is an arbitrary variation of $u, \delta a=a^{*}-a$ for an arbitrary function $a^{*}$ satisfying the constraint $0 \leqslant a^{*} \leqslant 1, \delta b=b^{*}-b$ for an arbitrary function $b^{*}$ satisfying the constraint $0 \leqslant b^{*} \leqslant 1, \delta \theta$ is an arbitrary variation of $\theta$ and $\delta \Lambda=\Lambda^{*}-\Lambda$ for an arbitrary non-positive real number $\Lambda^{*}$. Here, $a, b$ and $\theta$ are extended to the whole domain $\Omega$ from $\Omega_{\mathrm{d}}$ 'naturally' Assuming that

$$
\nu_{i}=\delta u_{i}+\frac{\partial u_{i}}{\partial a} \delta a+\frac{\partial u_{i}}{\partial b} \delta b+\frac{\partial u_{i}}{\partial \theta} \delta \theta \in H^{1}(\Omega), \quad i=1,2,
$$

the optimality criterion can be obtained as

$$
\begin{aligned}
& \sum_{i, j, k, l=1}^{2} \int_{\Omega} E_{i j k l}^{\mathrm{G}}(x) \frac{\partial u_{k}}{\partial x_{l}} \frac{\partial \nu_{i}}{\partial x_{j}} \mathrm{~d} \Omega+\sum_{i=1}^{2} \int_{I_{\mathrm{D}}} \lambda\left(u_{i}-g_{i}\right) \nu_{i} \mathrm{~d} \Gamma \\
& =\sum_{i=1}^{2} \int_{\Omega} f_{i} \nu_{i} \mathrm{~d} \Omega+\sum_{i=1}^{2} \int_{r_{\mathrm{T}}} t_{i} \nu_{i} \mathrm{~d} \Gamma \quad \forall \nu \in V, \\
& \int_{\Omega}\left\{\sum_{i, j, k, l=1}^{2} \frac{1}{2} \frac{\partial E_{i j k l}^{\mathrm{G}}}{\partial a} \frac{\partial u_{k}}{\partial x_{l}} \frac{\partial u_{i}}{\partial x_{j}}+\Lambda b\right\} \delta a \mathrm{~d} \Omega \geqslant 0 \\
& \quad \text { for every } \delta a=a^{*}-a, 0 \leqslant a^{*} \leqslant 1 \text { in } \Omega, \\
& \int_{\Omega}\left\{\sum_{i, j, k, l=1}^{2} \frac{1}{2} \frac{\partial E_{i j k l}^{\mathrm{G}}}{\partial b} \frac{\partial u_{k}}{\partial x_{l}} \frac{\partial u_{i}}{\partial x_{i}}+\Lambda a\right\} \delta b \mathrm{~d} \Omega \geqslant 0 \\
& \quad \text { for every } \delta b=b^{*}-b, 0 \leqslant b^{*} \leqslant 1 \text { in } \Omega, \\
& \int_{\Omega}\left\{\sum_{i, j, k, l=1}^{2} \frac{1}{2} \frac{\partial E_{i j k l}^{\mathrm{G}}}{\partial \theta} \frac{\partial u_{k}}{\partial x_{l}} \frac{\partial u_{i}}{\partial x_{j}}\right\} \delta \theta \mathrm{d} \Omega=0 \text { for every } \delta \theta, \\
& \left\{\int_{\Omega_{\mathrm{d}}}(1-a b) \mathrm{d} \Omega-\Omega_{\mathrm{s}}\right\} \delta \Lambda \geqslant 0 \text { for } \delta \Lambda=\Lambda^{*}-\Lambda, \Lambda^{*} \leqslant 0 .
\end{aligned}
$$

Noting that it has been assumed that $a, b, \theta \in H^{1}(\Omega)$, (11) may hold if $\partial u / \partial a, \partial u / \partial b$, $\partial u / \partial \theta \in H^{1}(\Omega)$. Since a small change of the microstructure yields a small change in the macroscopic homogenized elasticity tensor, and since its degree is much smaller than that of the microstructure itself, the effect on the displacement field of the 'porous' structure due to change of microstructure must be significantly small. That is, $\partial u / \partial a$ and others could be as smooth as $u$ itself, i.e., the assumption that $\partial u / \partial a, \partial u / \partial b, \partial u / \partial \theta \in H^{1}(\Omega)$ may be realistic.

Equation (12) is nothing but the form of the principle of virtual displacement. Inequality (13) can be equivalently expressed by

$$
a=\operatorname{Min}\left\{\operatorname{Max}\left\{0, a-\rho_{a}\left(\frac{1}{2} \sum_{i, j, k, l=1}^{2} \frac{\partial E_{i j k l}^{\mathrm{G}}}{\partial a} \frac{\partial u_{k}}{\partial x_{l}} \frac{\partial u_{i}}{\partial x_{j}}+\Lambda b\right)\right\}, 1\right\}
$$

for an arbitrary positive number $\rho_{a}$. The nonlinear equation (17) is obtained by using the fact that 


$$
\begin{aligned}
& u_{\min } \leqslant u \leqslant u_{\max }: \quad(u-f)(\nu-u) \geqslant 0 \quad \forall \nu \text { such that } u_{\min } \leqslant \nu \leqslant u_{\max } \\
& \Leftrightarrow u=\operatorname{Min}\left\{\operatorname{Max}\left\{u_{\min }, f\right\}, u_{\max }\right\} .
\end{aligned}
$$

Similarly, (14) can be expressed by

$$
b=\operatorname{Min}\left\{\operatorname{Max}\left\{0, b-\rho_{b}\left(\frac{1}{2} \sum_{l, j, k, l=1}^{2} \frac{\partial E_{i j k l}^{\mathrm{G}}}{\partial b} \frac{\partial u_{k}}{\partial x_{l}} \frac{\partial u_{i}}{\partial x_{j}}+\Lambda a\right)\right\}, 1\right\}
$$

for an arbitrary positive number $\rho_{b}$. Equation (15) yields

$$
\frac{1}{2} \sum_{i, j, k, l=1}^{2} \frac{\partial E_{i j k l}^{\mathbf{G}}}{\partial \theta} \frac{\partial u_{k}}{\partial x_{l}} \frac{\partial u_{i}}{\partial x_{j}}=0
$$

The Lagrange multiplier $\Lambda$ to the volume constraint satisfies

$$
\Lambda=\operatorname{Min}\left\{0, \Lambda-\rho_{\Lambda}\left(\int_{\Omega_{\mathrm{d}}}(1-a b) \mathrm{d} \Omega-\Omega_{\mathrm{s}}\right)\right\}
$$

for an arbitrary positive number $\rho_{A}$.

It is clear that (17)-(20) suggest a solution method for the optimization problem (7).

\section{An optimality criteria method}

The condition of the optimality criteria derived in the previous section suggests a solution method of the optimization problem (7). More precisely, an iterative scheme is easily obtained as follows:

Step 1. Assume appropriate initial values of $a^{(0)}, b^{(0)}, \theta^{(0)}$ and $\Lambda^{(0)}$, and set $k=0$. $k \leftarrow k+1$

Step 2. Compute the rotated homogenized elasticity tensor $\mathbb{E}^{\mathrm{G}(k)}$.

Step 3. Obtain the displacement field $u^{(k)}$ satisfying the principle of virtual displacement (4), i.e., one of the optimality criteria (12) for the elasticity tensor $\mathbb{E}^{\mathrm{G}(k)}$ computed in Step 2.

Step 4. Update the values of $a, b, \theta$ and $\Lambda$, i.e., obtain $a^{(k)}, b^{(k)}, \theta^{(k)}$ and $\Lambda^{(k)}$ using $u^{(k)}$ and the optimality criteriia $(17),(18),(19)$ and $(20)$.

As mentioned above, the homogenized elasticity tensor $\mathbb{E}^{\mathrm{H}}$ is obtained for a set of discrete values of $a$ and $b$, and it is assumed by an appropriate interpolation function to obtain a form of continuous function of $a$ and $b$. This makes it possible to avoid solving the homogenization problem for arbitrary values of $a$ and $b$ 'everywhere' in the design domain $\Omega_{\mathrm{d}}$. In other words, the homogenization problem is solved for certain sizes of holes prior to the optimization problem. Using the interpolated homogenized elasticity tensor $\mathbb{E}^{\mathrm{H}}$ and the rotation $\theta$, the 
rotated homogenized elasticity tensor $\mathbb{E}^{\mathrm{G}}$ is computed. It is noted that the homogenization problem (1) is solved by applying a finite element method. In the present work, four-node quadrilateral isoparametric elements are used. The principle of virtual displacement (14) is similarly discretized using four-node quadrilateral isoparametric elements, and finite element approximations of the displacement field $u$ are obtained. Since a very standard finite element method is applied to solve these problems, it is unnecessary to provide its details.

There are many ways to update the design variables $a, b$ and $\theta$, which are discretized by using a finite element model for solving the principle of virtual displacement, and the Lagrange multiplier $\Lambda$. Although the design variables can be discretized at the Gaussian integration points to form the stiffness matrix, we shall discretize these using the values at the center of each finite element, i.e., $a, b$ and $\theta$ are approximated by piecewise constant functions, since the discretization at Gaussian points brings inaccuracy in computing the element stiffness matrices. That is, while discretization at each Gaussian point has the advantage that it can handle 4 times more design variables with the same finite element $m: s s$, the element whose material property is not uniform cannot integrate accurately by a Gaussian integration and causes instability in convergence. Since we shall discretize the design domain in which a structure is placed using very refined finite elements, the number of discrete design variables becomes very large. Because of this, we are solving the optimization problem using the optimality criteria method rather than applying a mathematical programming method available in standard codes for optimization.

The simplest updating scheme of $a, b, \theta$ and $\Lambda$ is obtained by direct application of the expression of the optimality criteria, (17), (18), (19) and (20). More precisely,

$$
\begin{aligned}
& a^{(k)}=\operatorname{Min}\left\{\operatorname{Max}\left\{0, a^{(k-1)}-\rho_{a}^{(k)}\left(\frac{1}{2} \sum_{i, j, k, l=1}^{2} \frac{\partial E_{i j k l}^{\mathrm{G}(k)}}{\partial a} \frac{\partial u_{k}^{(k)}}{\partial x_{l}} \frac{\partial u_{i}^{(k)}}{\partial x_{j}}+\Lambda^{(k-1)} b^{(k-1)}\right)\right\}, 1\right\} \\
& b^{(k)}=\operatorname{Min}\left\{\operatorname{Max}\left\{0, b^{(k-1)}-\rho_{b}^{(k)}\left(\frac{1}{2} \sum_{i, j, k, l=1}^{2} \frac{\partial E_{i j k l}^{\mathrm{G}(k)}}{\partial b} \frac{\partial u_{k}^{(k)}}{\partial x_{l}} \frac{\partial u_{i}^{(k)}}{\partial x_{j}}+\Lambda^{(k-1)} a^{(k)}\right)\right\}, 1\right\} \\
& \theta^{(k)}=\theta^{(k-1)}-\rho_{\theta}^{(k)} \frac{1}{2} \sum_{i, j, k, l=1}^{2} \frac{\partial E_{i j k l}^{\mathrm{G}(k)}}{\partial \theta} \frac{\partial u_{k}^{(k)}}{\partial x_{l}} \frac{\partial u_{i}^{(k)}}{\partial x_{j}} \\
& \Lambda^{(k)}=\operatorname{Min}\left\{0, \Lambda^{(k-1)}-\rho_{\Lambda}^{(k)}\left(\int_{\Omega_{\mathrm{d}}}\left(1-a^{(k)} b^{(k)}\right) \mathrm{d} \Omega-\Omega_{\mathrm{s}}\right)\right\}
\end{aligned}
$$

for arbitrary but appropriate positive numbers $\rho_{a}^{(k)}, \rho_{b}^{(k)}, \rho_{\theta}^{(k)}$ and $\rho_{A}^{(k)}$. It is expected that for sufficiently small numbers, the above iterative scheme is convergent as $k$ goes to infinity, but it could be very slow. Thus, some modifications must be implemented to be practical.

The first modification is for the design variables $a$ and $b$ as well as the Lagrange multiplier A. Defining

$$
D_{a}^{(k)}=-\left(\Lambda^{(k)} b^{(k-1)}\right)^{-1} \sum_{i, j, k, l=1}^{2} \frac{1}{2} \frac{\partial E_{i j k l}^{\mathrm{G}(k)}}{\partial a} \frac{\partial u_{k}^{(k)}}{\partial x_{l}} \frac{\partial u_{i}^{(k)}}{\partial x_{j}}
$$

the design variable $a$ is updated by 


$$
\begin{aligned}
& a^{(k)}= \\
& \begin{cases}\operatorname{Max}\left\{(1-\zeta) a^{(k-1)}, 0\right\} & \text { if } a^{(k-1)}\left(D_{a}^{(k)}\right)^{\eta} \leqslant \max \left\{(1-\zeta) a^{(k-1)}, 0\right\}, \\
a^{(k-1)}\left(D_{a}^{(k)}\right)^{\eta} & \text { if } \operatorname{Max}\left\{(1-\zeta) a^{(k-1)}, 0\right\} \leqslant a^{(k-1)}\left(D_{a}^{(k)}\right)^{\eta} \leqslant \operatorname{Min}\left\{(1+\zeta) a^{(k-1)}, 1\right\}, \\
\operatorname{Min}\left\{(1+\zeta) a^{(k-1)}, 1\right\} & \text { if } \operatorname{Min}\left\{(1+\zeta) a^{(k-1)}, 1\right\} \leqslant a^{(k-1)}\left(D_{a}^{(k)}\right)^{\eta},\end{cases}
\end{aligned}
$$

where $\zeta$ is a move limit and $\eta$ is a weighing factor. The iteration scheme (24) is a slight modification of the simple iterative method $(22)$ for the special case that

$$
\rho_{a}^{(k)}=a^{(k-1)} / \Lambda^{(k)} b^{(k-1)}
$$

Here the Lagrange multiplier $\Lambda^{(k)}$ to the volume constraint must be known to update the design variable $a$. As such, the value of the Lagrange multiplier $\Lambda$ is computed by the bisection method in an inner loop:

$$
\begin{aligned}
& \text { Substep 1. Set up }-\Lambda_{\min }^{(k, 0)} \text { so that }-\Omega_{\mathrm{s}}^{(k)}\left(\Lambda_{\min }^{(k, 0)}\right)<\Omega_{\mathrm{s}} \\
& \text { and }-\Lambda_{\max }^{(k, 0)} \text { so that }-\Omega_{\mathrm{s}}^{(k)}\left(\Lambda_{\max }^{(k, 0)}\right)>\Omega_{\mathrm{s}} \\
& \\
& m=0
\end{aligned}
$$

where

$$
\Omega_{\mathrm{s}}^{(k)}(\Lambda)=\int_{\Omega_{\mathrm{d}}}\left(1-a^{(k)}(\Lambda) b^{(k)}(\Lambda)\right) \mathrm{d} \Omega
$$

The design variable $b$ is updated in exactly the same way as $a$.

The second modification is for the angle of rotation $\theta$ of the unit cell. To this end, we recall that the original goal is to maximize the total potential energy by finding appropriate rotation of the microstructure characterized by rectangular holes. In other words, we intend to find the angle of rotation $\theta$ satisfying the necessary condition $\partial \Pi_{\lambda} / \partial \theta=0$. Noting that

$$
\begin{aligned}
\Pi_{\lambda}(u)= & \frac{1}{2} \sum_{i, j, k, l=1}^{2} \int_{\Omega} C_{i j k l}^{\mathrm{G}} \sigma_{k l} \sigma_{i j} \mathrm{~d} \Omega+\frac{1}{2} \sum_{i, j, k=1}^{2} \int_{\Gamma_{\mathrm{D}}} \frac{1}{\lambda} \sigma_{i j} n_{j} \sigma_{i k} n_{k} \mathrm{~d} \Gamma \\
& -\sum_{i, j=1}^{2} \int_{\Gamma_{\mathrm{D}}} g_{i} \sigma_{i j} n_{j} \mathrm{~d} \Gamma,
\end{aligned}
$$

where $\boldsymbol{\sigma}$ is the stress tensor at equilibrium and $\mathbb{C}^{\mathbf{G}}$ is the rotated homogenized compliance tensor, it is possible to find $\theta$ satisfying the necessary condition using the right-hand side of (27). Since the penalty parameter $\lambda$ and the constrained displacement $g$ are independent to the microstructure, its dependence on $\theta$ must vanish. Thus, the appropriate $\theta$ can be obtained by examining the first term on the right-hand side of (27). Following Pedersen [22] and also 
Gibiansky and Chercaev [23], such a $\theta$ can be identified with the principal direction of the stress tensor $\sigma$ for the case of 'shear weak type materials'. Thus, computing the principal stresses as well as the angle of the principal direction using the displacement field $u^{(k)}$, an updating scheme of the angle of rotation $\theta^{(k)}$ is obtained.

\section{A verification of the present method}

We shall examine the present method to solve the optimization problem (7) by solving a simple problem whose solution may be obtained analytically using a simple structural model such as a truss or a beam. A two-bar frame structure as shown in Fig. 3 is considered.

For the fixed values of the applied load $P$ and the horizontal length $L$ of the frames, the optimal height $H$ is determined by minimizing the mean compliance. If the cross section of the frames is rectangular with the unit width, the optimal height $H$ is obtained as $H=2 L$ if the total volume of the frames is assumed to be constant, if this structure is assumed to be a truss. Defining a rectangular design domain $\Omega_{\mathrm{d}}$ that is larger than the size $L \times 2 L$, where $L=10 \mathrm{~cm}$, the generalized layout problem (7) is solved by discretizing $\Omega=\Omega_{\mathrm{d}}$ using $40 \times 96$ finite elements. The homogenized elasticity tensor $\mathbb{E}^{\mathrm{H}}$ applied here is computed at $6 \times 6$ sampling points in the design variables $a$ and $b$, respectively, and is interpolated by the Bezier fuictions. The homogenization problem (1) is solved using $10 \times 10$ finite elements for the 'solid' isotropic material characterized by Young's modulus $E=100 \mathrm{GPa}$ and Poisson's ratio $\nu=0.3$. As shown in Fig. 4, the present method forms a two-bar frame structure as the amount of the 'solid' material is decreased, and the solutions converge to the theoretical solution obtained by assuming beam bending and axial deformation. It is also noted that the present method can not only provide the basic two-bar frame structure but also the size of the cross section of bars, especially for the case of large volume of the 'solid' material.

The same problem is also solved by using different finite elements, i.e., using three node triangular elements. As shown in Fig. 5, the optimal solution obtained is very similar to the one computed by using four-node quadrilateral elements. Thus, at least for this example, the choice of specific finite elements is not so critical.

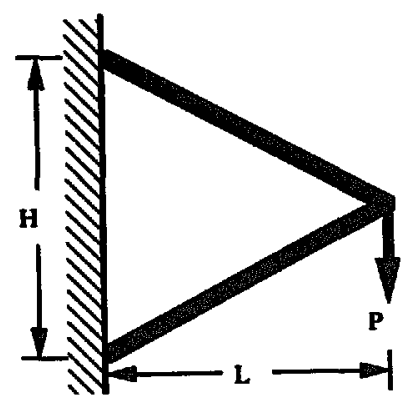

Fig. 3. A two-bar frame structure. 

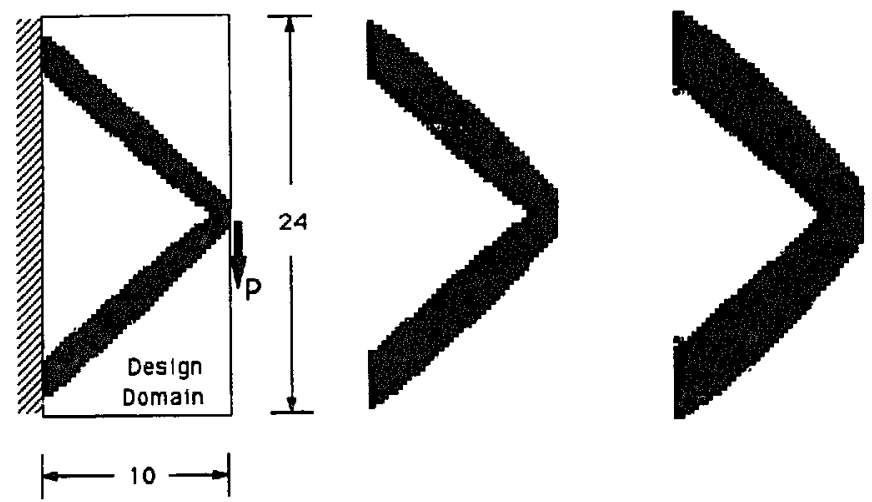

Fig. 4. Optimal size and configuration of a two-bar frame structure.

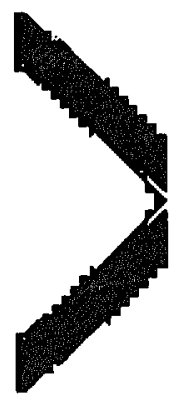

Fig. 5. Optimal configuration by triangular elements.

\section{Convergence property of the finite element approximation}

The next verification of the present method is whether the shape and topology, i.e., the configuration of the structure obtained as the optimum converges to the unique one as finite element meshes are uniformly refined, while other conditions are fixed. To check this convergence property, let us solve the optimization problem (7) for a short cantilever subject to the vertical force at the free end, see Fig. 6. For three different volumes 80,60 and 40 of

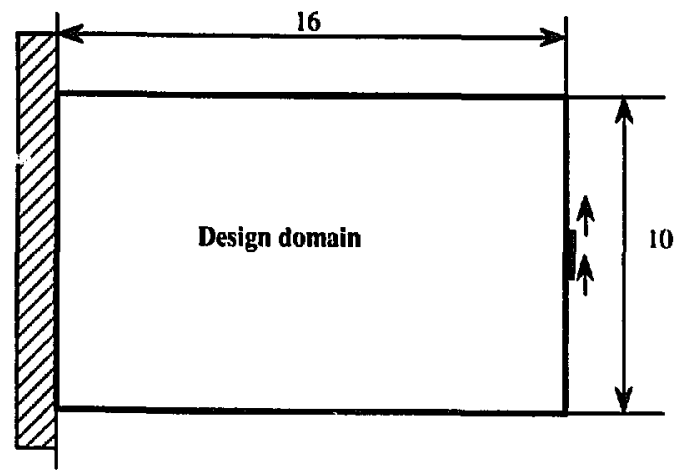

Fig. 6. Design domain for bending of a short cantilever. 

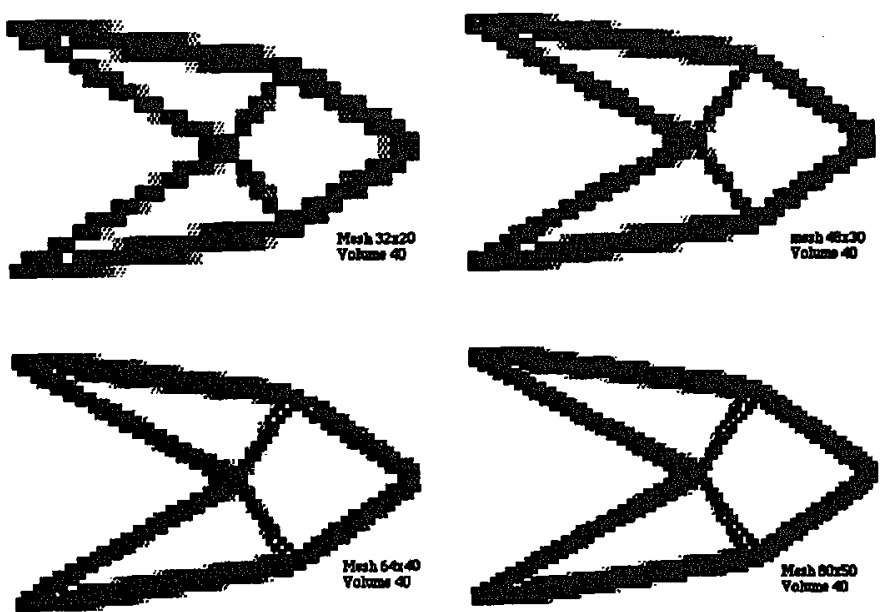

Fig. 7. Convergence of the optimal configuration $\left(\Omega_{d}=40\right)$.
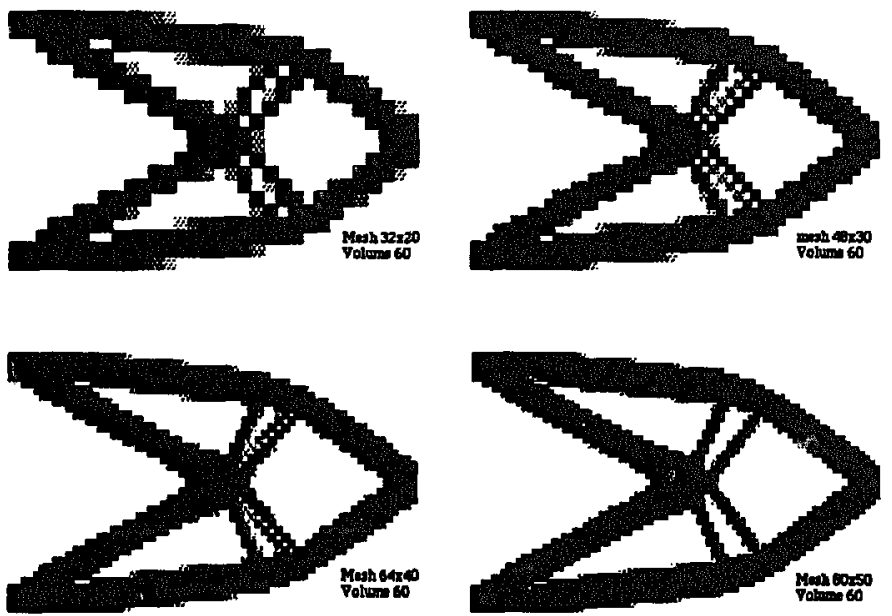

Fig. 8. Convergence of the optimal configuration $\left(\Omega_{d}=60\right)$.
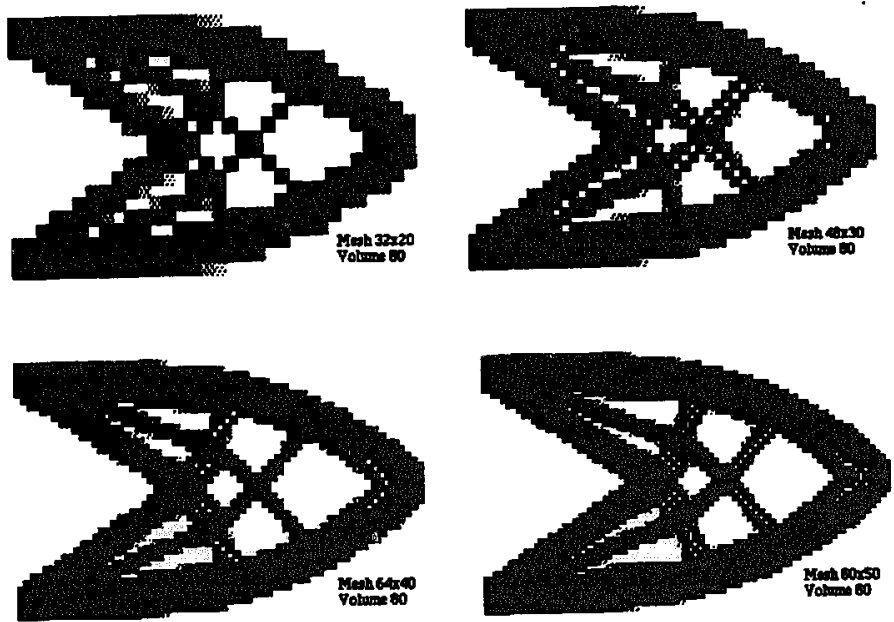

Fig. 9. Convergence of the optimal configuration $\left(\Omega_{d}=80\right)$. 


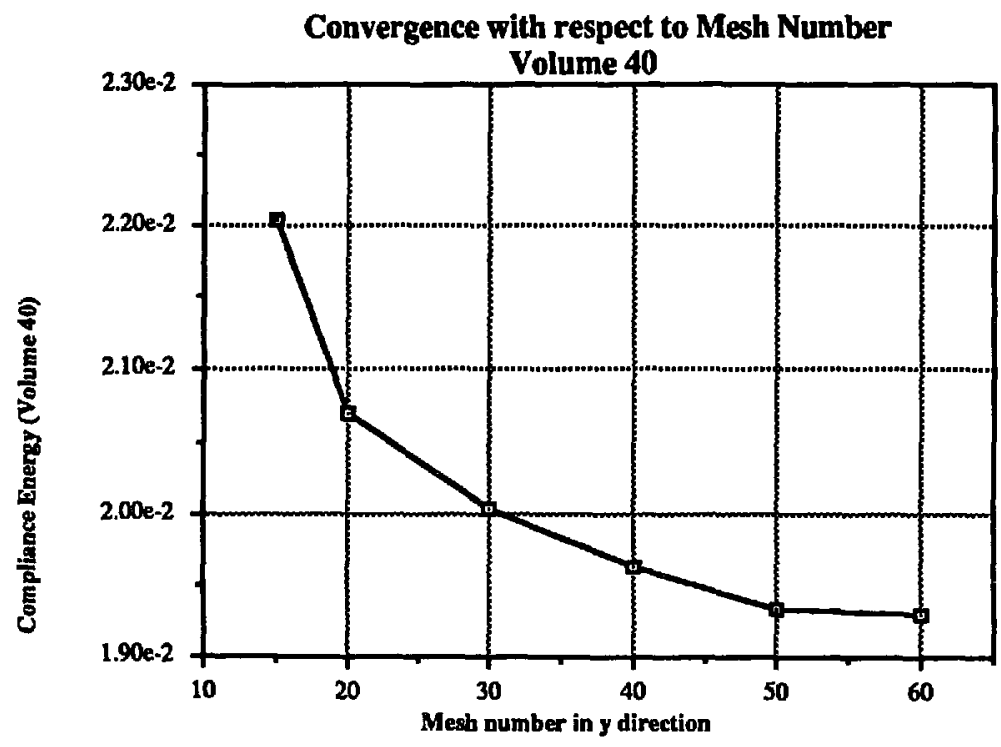

Convergence with respect to Mesh Number
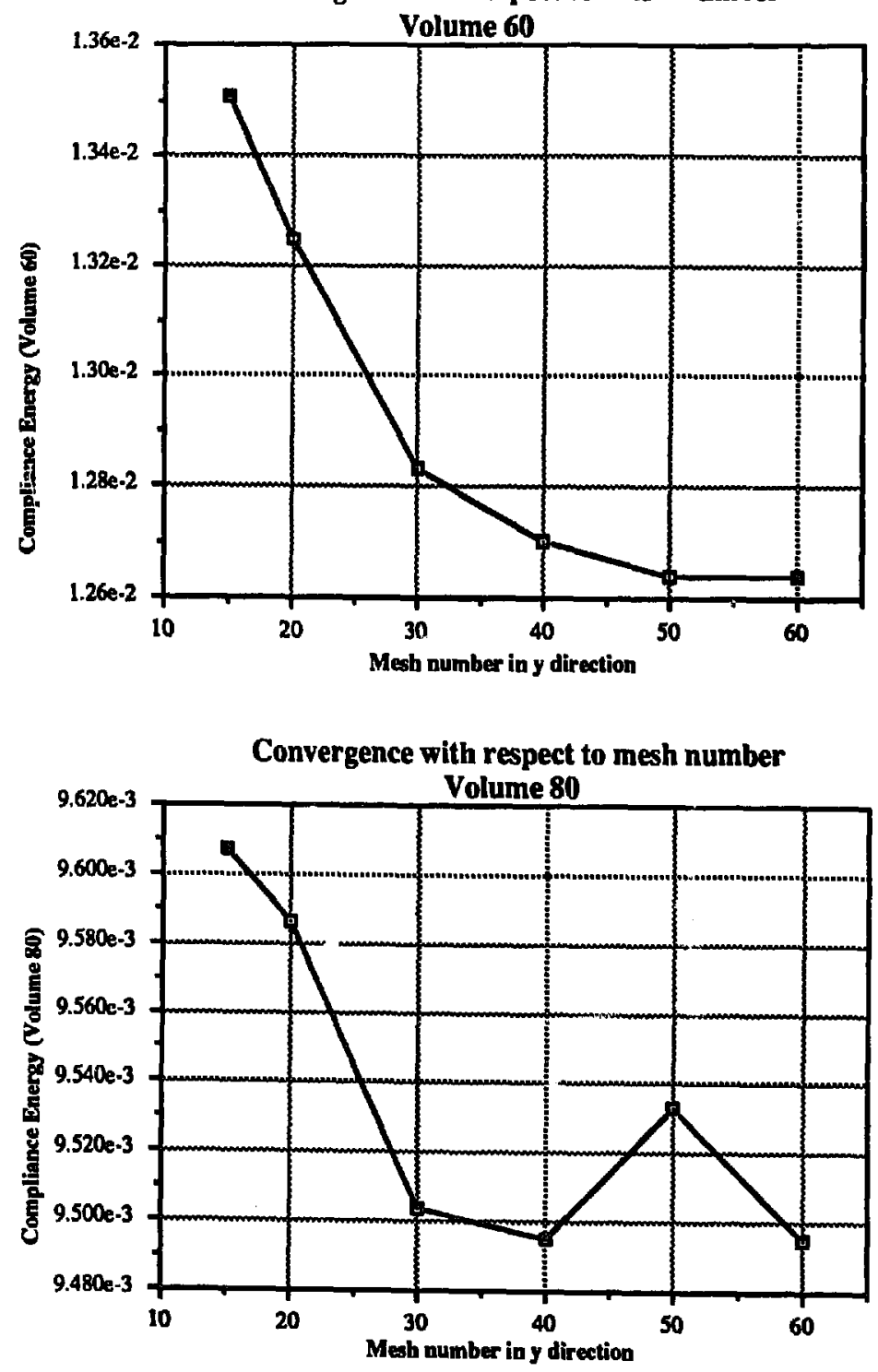

Fig. 10. Convergence of the mean compliance. 
the 'solid' material, the generalized layout problem (7) is solved by using $32 \times 20,48 \times 30$, $64 \times 40$ and $80 \times 50$ equal size finite elements covering a rectangular design domain $\Omega_{\mathrm{d}}$. Applying the same homogenized elasticity tensor to the first example, the optimal configurations are obtained as shown in Figs. 7,8 and 9. It is clear that the optimal configurations are convergent as the size of finite elements is reduced. Even every coarse mesh can provide a sufficient idea for the topology and shape of the optimal structure. If the present approach is used to find a rough idea of the optimal structure, it is not necessary to solve the problem using very refined meshes. After obtaining a rough configuration, it is possible to solve the problem using existing shape optimization codes which are commercially available and more capable to input various design constraints and to minimize other objective functions. Although the existing capability on the shape optimization is very limited for the topological change of the configuration of a structure, it can provide details of the shape of the structure.

Figure 10 presents convergence of the mean compliance as the size of finite element goes to zero. Despite non-monotonic convergence is observed for the case of $\Omega_{d}=80$, the mean compliance is steadily decreased as the size of finite elements is decreased.

Another interesting observation in the results obtained is that very truss-like frame structures are built if the amount of the 'solid' material is considerably smaller than that of the design domain. Each frame member is clearly straight. If the amount of the 'solid' material becomes large, the optimal design diverges from truss-like structures. Curved frames are generated, and more continuum-like shapes are formed. It is also noted that the topology of the optimal structure changes as the amount of the 'solid' material changes. Nine holes are generated for $\Omega_{\mathrm{s}}=80$, while four holes are formed for $\Omega_{\mathrm{s}}=40$ and 60 .

\section{Construction of the Michell truss}

The third verification of the homogenization method for the generalized layout problem is whether it can reproduce the Michell truss (Fig. 11), which is already known as the optimal truss structure for bending and which is solved analytically as the most typical problem of structural layout, see [27]. To do this, the setting given in Fig. 12 is assumed. That is, a bending force $P$ must be optimally transferred to the circular fixed support by constructing a truss-like structure. Using $110 \times 80$ uniform finite elements to discretize a rectangular design domain $\Omega=\Omega_{\mathrm{d}}$, the generalized layout problem (7) is solved by approximately setting the

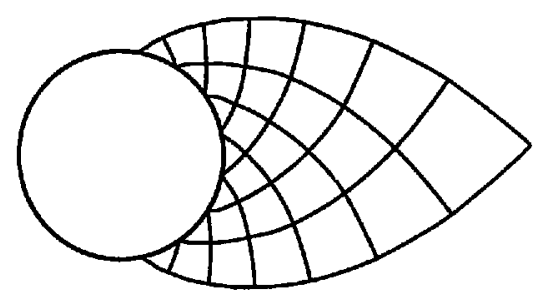

Fig. 11. Analytical solution of the Michell truss.

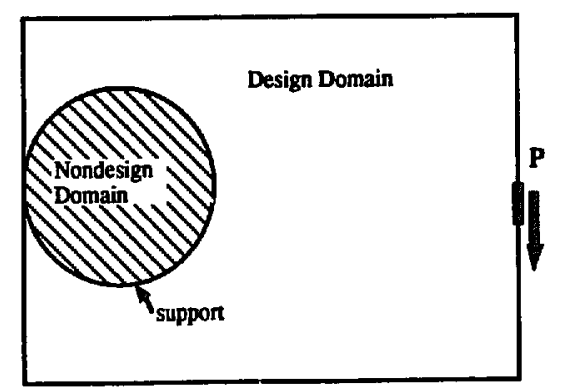

Fig. 12. Setting of the problem similar to the Michell truss. 
circular rigid support using rectangular finite elements whose design variances are fixed as $a=b=\theta=0$. The degrees of freedom on the boundary of the support are also fixed to have no deformation inside of the 'support'. The approximation made here to represent the circular support may be too crude in order to study details of layout near the support. However, our interest is to reproduce the Michell truss-like structure in the global sense that approximate configurations are examined. Figure 13 shows the optimal layout for various volumes of the 'solid' material. It is clear that the present method can generate Michell truss-like structures, i.e., frames generated are mutually orthonormal in most of the domain. Note that the 'frames' are rigidly connected at each junction, while hinge joints are generally assumed in analytical study of the Michell truss.

An interesting observation is the similar structures are repeatedly utilized with different sizes to form the optimal structure. Since the structure must be built up for bending, the size of most of the outer members is the largest. If straight bar frames are assumed, it is possible to determine their optimal size by lumping the 'porous' structure to a solid one from the results obtained by the present method. It is also believed that 'porous' frames are generated because of the size restriction of finite element meshes. If much refined finite element discretization is applied, 'porous' frames would be eliminated and solid frames would be generated for this type of simple structures. In this sense, the present method can not only deal with classical layout problems of frames but also with sizing problems for frames at the same time. In this respect, the method proposed here can solve the generalized layout problem which involves topology, shape and sizes of a structure.

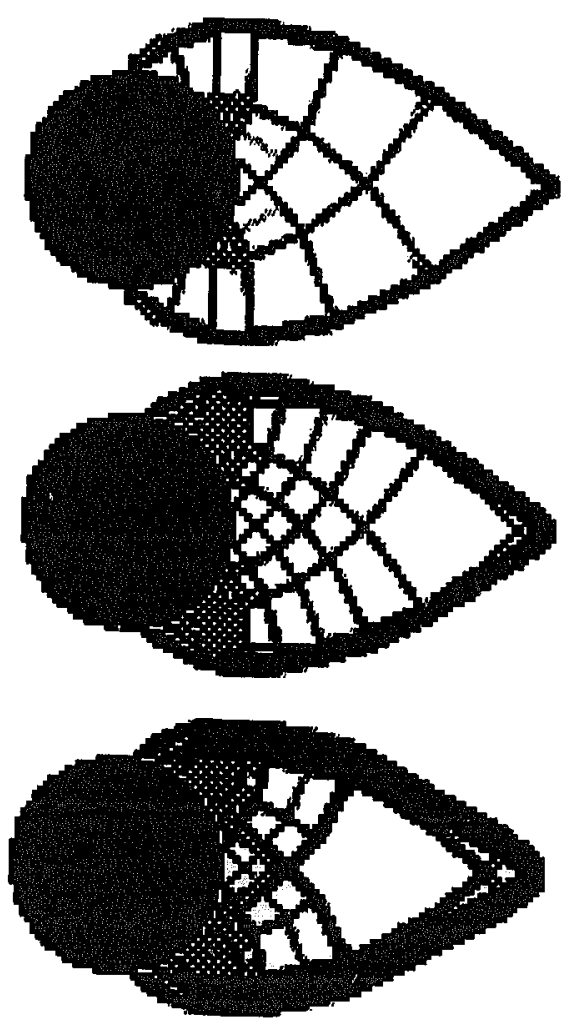

Fig. 13. Optimal configurations for different volumes of the 'solid'. 
It is also noteworthy that while an analytical solution of the Michell truss should have an infinite number of bars, our solutions have a finite number of bars. These results are because of rather concentrating tendency of our material constants. This tendency appears again in later examples, for example in Fig. 19 it becomes fiber-like rather than uniform distribution. Also in Figs. 32 and 33 the porous type area is checker-flag type material distribution rather than uniform. This tendency cannot appear if thickness is used as design variables in which material constants have a linear relation to design variables. Also this tendency does not appear if fiber density is used as design variables.

\section{Effect of the boundary condition}

Next we shall examine the effects of different boundary conditions using a short beam used in the example for convergence study. Instead of applying a transverse force that yields bending to the beam, a tensile traction is applied on the middle one-third portion of the free end of the beam as shown in Fig. 14. Using the same finite element model and the unit cell model used in Section 6, the generalized layout problem (7) is solved.

The optimal layout is obtained as shown in Fig. 15. In this case, the fixed support condition is not extended along the whole left edge although the fixed boundary condition is assumed on the whole edge in stress analysis for the 'porous' structure. This means that the assumption of a larger boundary for the fixed support would not affect the optimal configuration, i.e., the optimal structure defines necessary range of the boundary for the fixed support condition. As shown in Fig. 15, only a small portion of the left edge is used as the fixed condition, since the applied tensile force can be effectively supported by straight bars in which the axial deformation and stress are dominant. Two solid bars are formed in the left side to carry out the tensile force while porous medium is generated in the vicinity of the edge on which tensile traction is applied. Porous medium is generated in order to diverse stresses due to the applied traction. It is noted that if the support condition on the left edge is roller type instead of the fixed support, the optimal design would become an accumulation of many thin straight solid bars, the total volume of which is exactly equal to the given 'solid' material volume, as illustrated in Fig. 16.

It is also noted that there are many interpretations of the porous medium generated.

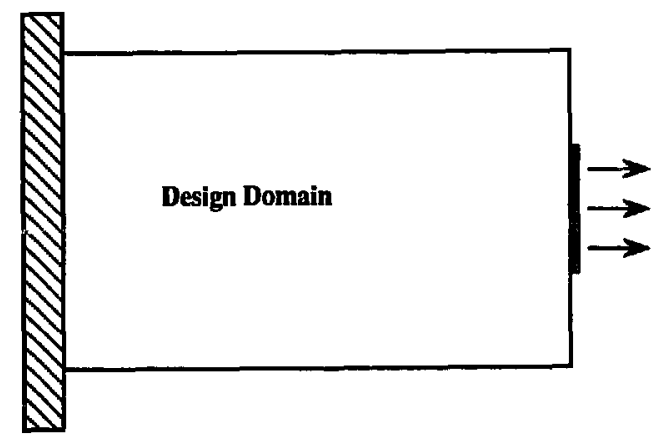

Fig. 14. Design domain with a tensile traction applied.

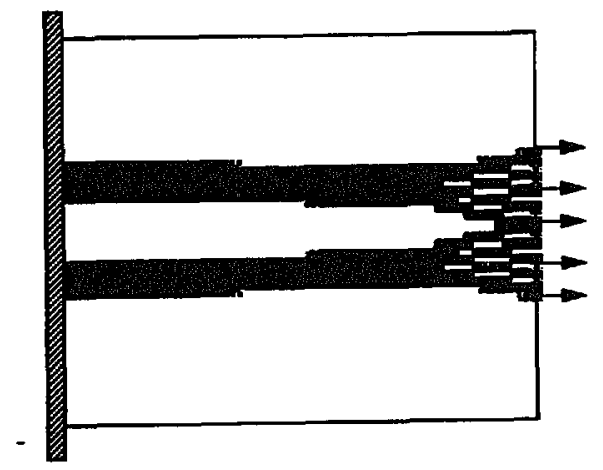

Fig. 15. Optimal design for the fixed boundary condition. 


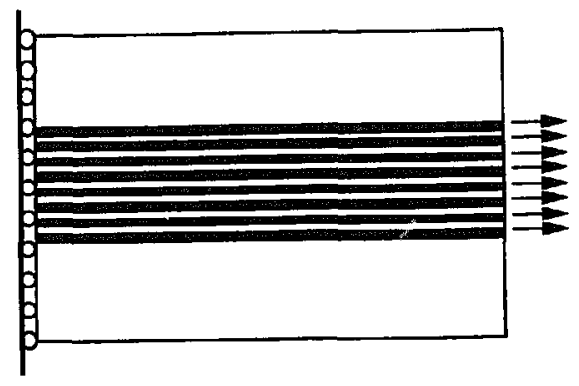

Fig. 16. Illustration of the optimal design for the roller support condition.
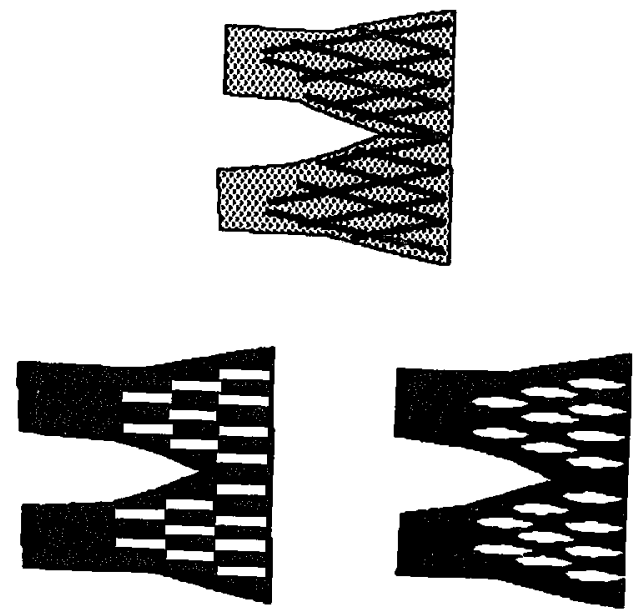

Fig. 17. Possible equivalent design of the right end part.

Although square micro scale voids are assumed in the present method, the computed result suggests that the designs given in Fig. 17 may be equally strong to transmit the applied traction since complete 'solid' and 'void' distributions are staggered.

In order to show that the optimal structure strongly depends on the boundary condition, we shall consider the same design domain and the tensile traction of the right edge, but, instead of the fixed support condition, we shall apply the uniformly distributed traction on the left edge so that the structure can be equilibrium within rigid body motions as shown in Fig. 18.

Assuming that the volume of 'solid' material is $25 \%$ of that of the design domain $\Omega_{d}$, the generalized layout problem (7) yields the optimal distribution of the 'solid' material as shown in Fig. 19. The optimal structure consists of very many smoothly curved thin porous bars which are 'knitted' by much thinner curved bars to reinforce in the transverse direction. Although they are not orthogonal, they are smoothly connected. Since linear elasticity is assumed in this program, the same result is expected for the compressible traction. In this case, it is necessary to consider the possibility of local buckling of thin bars in order to obtain the 'true' optimum structure. Figure 20 shows a net type frame structure suggested by the optimal solution given in Fig. 19.

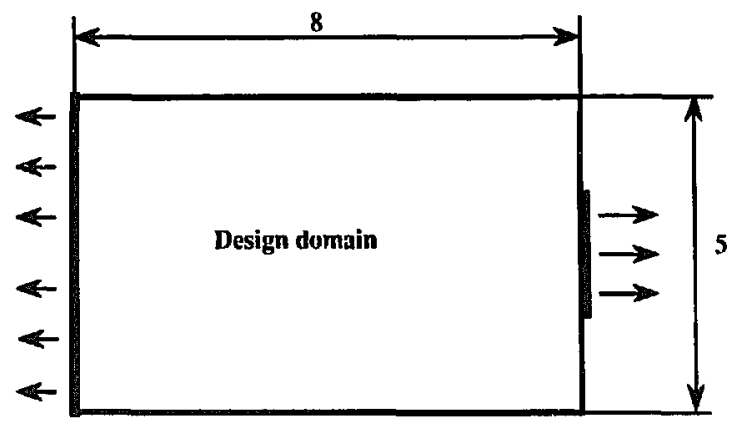

Fig. 18. Design domain without the support condition.

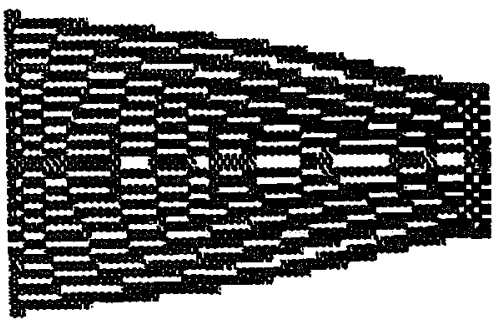

Fig. 19. Distribution of the 'solid' materials. 


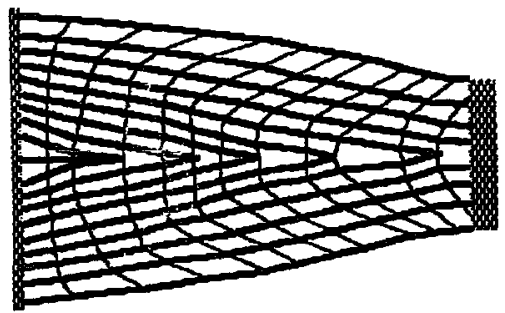

Fig. 20. 'Idealized' net type framed structure.

\section{Effect of undesigned region}

The next application of the homogenization method in the generalized layout problem involves the fillet design to transfer a tensile force from the narrow right edge to the wider left edge as shown in Fig. 21. Two cases are considered to examine effect of the area of undesigned domain $\boldsymbol{\Omega}-\boldsymbol{\Omega}_{\mathrm{d}}$. It is also noted that the first case of the present example differs from the study of the last problem in the previous example. Here, the traction is applied on the undesigned region, whereas it is directly applied on the edge of the design domain $\Omega_{\mathrm{d}}$ in the previous example. Thus the result obtained should be different from that of the previous case.

The biack portion in Fig. 21 is the undesigned domain, while the white portion is the design domain in which 'solid' material, i.e., equivalently microscale voids are distributed. Assigning three different 'solid' material volumes in each case, the optimal layout problem (7) is solved. As shown in Fig. 22, very discrete structures are placed in the design domains to transfer the applied tensile force except the case that a porous medium is generated for the largest volume with a thin undesigned domain. If the undesigned portion is thin, two symmetric bars are generated together with an reinforcement in the transverse direction with an extremely thin (or porous) bar. If the undesigned portion becomes thick enough, only one bar structure is formed. It should be noted that no matter how refined meshes are applied in the vicinity of the interface of the undesigned and designed domains for the case of thick undesigned region,
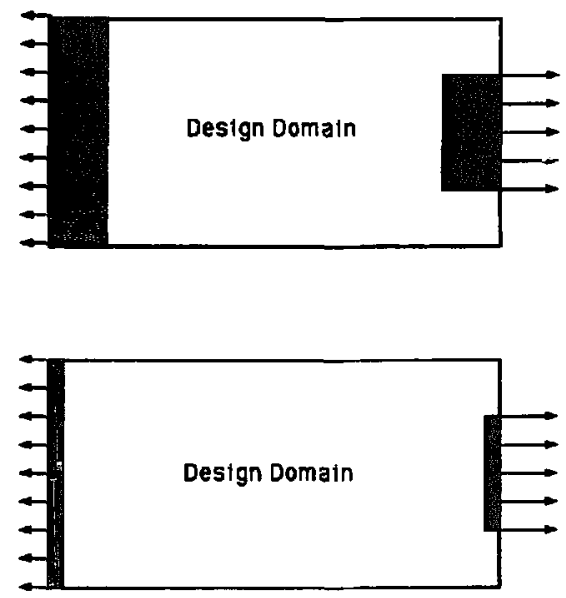

Fig. 21. Two different design domains for the fillet design. 

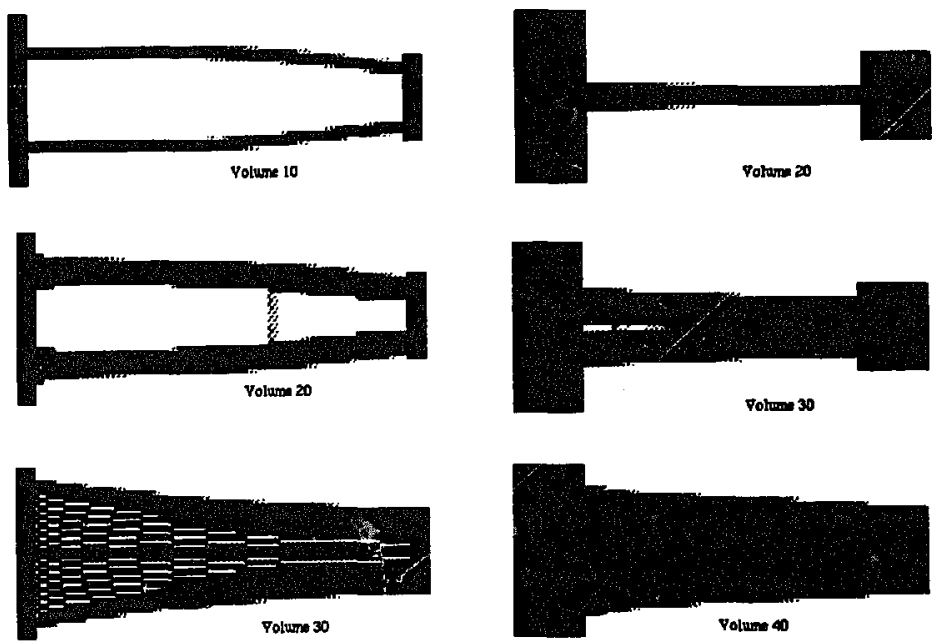

Fig. 22. Optimal configurations for the fillet design.

very smooth shape cannot be obtained from the formulation (7), since the mean compliance is used as the objective function which may not be able to deal with local values of stresses, i.e., rounding arcs are not generated at the corners as a typical optimal shape of the fillet problem. To obtain a similar result by the boundary variation method for the shape optimization problem, the objective function must be replaced by, for example, the maximum equivalent stress. We shall retain this extension for future works. Despite this limitation, the present approach using the mean compliance can determine an overall structural configuration.

\section{Restriction on the design domain}

This example is for the case that there is some area where 'solid' material cannot be placed because of plumbing, wiring, walls or other obstacles. The setting in Fig. 23 involves that the

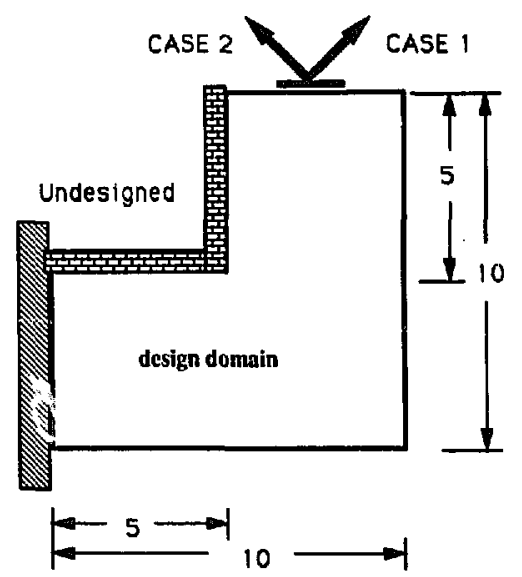

Fig. 23. Layout in an L-shaped domain for two different loadings. 


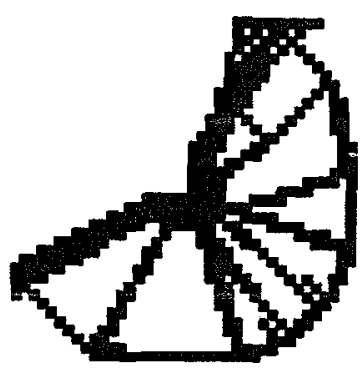

Fig. 24. Optimal configuration for Case $1\left(\Omega_{\mathrm{s}}=\right.$ $20 \mathrm{~cm}^{2}$ ).

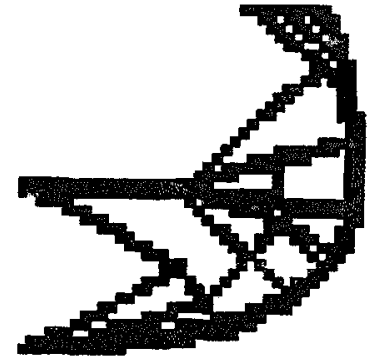

Fig. 25. Optimal configuration for Case $2\left(\Omega_{4}=\right.$ $20 \mathrm{~cm}^{2}$ ).

upper left corner portion is not usable for design. Two different loads are applied on the center portion of the top surface, and the left side of the L-shaped domain is fixed. The 'solid' material volume is $20 \mathrm{~cm}^{2}$ while the volume of the design domain $\Omega_{\mathrm{d}}$ is $75 \mathrm{~cm}^{2}$.

Figure 24 shows the optimal configuration of the structure for Case 1 in which the applied traction acts as a tensile force to form the optimal structure. Since the traction force is inclined $45^{\circ}$, the fundamental design is a bar structure in the $45^{\circ}$ direction which is the best for axial tension. If there is no design restriction, such a tension bar can be formed. However, because of the restriction in the design domain, such a straight tension bar cannot be formed. Thus, at the corner point of the L-shaped domain, 'solid' material is distributed circularly. This then yields an arc beam to reinforce to sustain the bending load due to the 'bent' solid bar at the corner. Furthermore, thin bars appear to connect the arc beam to the main 'bent' solid bar, that is similar to a bicycle wheel.

On the other hand, if the traction force is applied as in Case 2, it acts as a bending force to the domain. Thus, the optimal structure is formed to be the best for bending, especially in the lower portion of the L-shaped design domain as shown in Fig. 25. That is, the lower part of the optimal configuration should be similar to the case for pure bending as in the example solved in Section 6. Since the traction is distributed on the center one-third portion of the top surface of the L-shaped domain, porous medium is generated as in the case of the pure tensile case studied in Section 8. A bar inclined by $135^{\circ}$ is formed to resist to the tensile traction in the upper portion of the domain, and it is supported by two thinner bars starting from the corner point of the L-shaped domain. Such a bar for tension is then connected to the lower portion along the right wall of the domain, and the tension force in the upper domain now becomes a bending force for the lower portion. It is noted that a rather solid medium is formed in the vicinity of the applied traction in Case 1 , while porous medium is generated in Case 2 . This could be explained as the solid medium must be placed since the arc reinforcement in Case 1 starts from right under the loading portion, whereas a bar supporting the tension bar is connected far down from the loading portion in Case 2.

\section{Transition from shape to topology optimization}

So far only rectangular finite elements are applied to solve the optimal design problem (7). Now let us examine the capability to use non-rectangular quadrilateral 4-node elements. To 


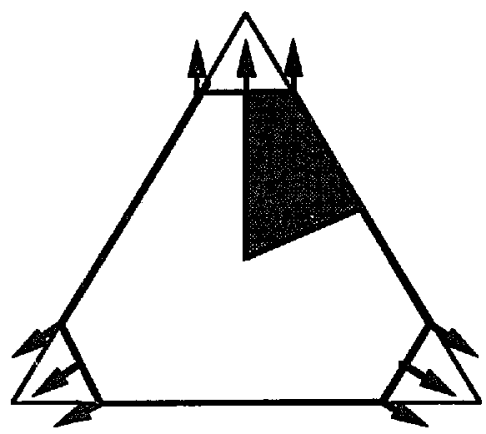

Fig. 26. Non-rectangular design domain.

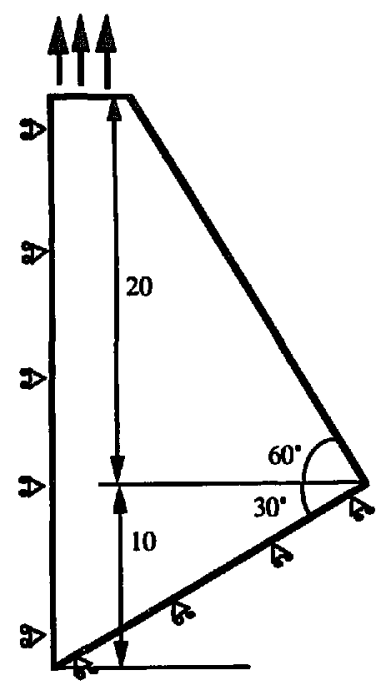

Fig. 27. Design domain for the optimization (1/6 portion of the original domain).

demonstrate it a 'triangular' design domain is considered as shown in Fig. 26. Applying the symmetry condition, only $1 / 6$ part of the design domain is modeled by the finite element method. The boundary condition and physical dimensions are specified in Fig. 27. The domain is discretized by $40 \times 40$ meshes which are not orthogonal. Using this example, we shall also examine the transition from the shape optimization to the topology optimization that forms more or less truss-like structures. To do this, the volume of the 'solid' material is varied in a wide range from $50 \mathrm{~cm}^{2}$ to $180 \mathrm{~cm}^{2}$, while the area of the $1 / 6$ of the original domain is $317.5 \mathrm{~cm}^{2}$.

As shown in Fig. 28, if there is a sufficient amount of 'solid' material, the optimal structure is singly connected without having holes inside the domain. The obtained shape is also very smooth, that is, of almost the same quality as the one obtained by the boundary variation method which is traditionally applied to solve the shape optimization problem. When the amount of 'solid' material volume is reduced, holes start appearing interior of the domain. It is also noted that the loading portion becomes porous when the amount of 'solid' material is really small in order to diverse the applied traction uniformly in all the directions equally, while the three compressed link bars in $30^{\circ}, 150^{\circ}$ and $270^{\circ}$ directions, are also porous. Thus, if these porous bars are replaced by solid bars maintaining the 'solid' material volume, the size of bars becomes very small. Thin solid bars which are in tension, are allocated in $90^{\circ}, 210^{\circ}$ and $330^{\circ}$ directions in the optimal structure. The outer frames are completely solid.

Transition from the singly connected domain to the multi-connected domain occurs at $\Omega_{\mathrm{s}}=130 \mathrm{~cm}^{2}-140 \mathrm{~cm}^{2}$. To examine details of the transition, larger but detailed material distribution is presented in Figs. 29 and 30 for $\Omega_{\mathrm{s}}=130 \mathrm{~cm}^{2}$ and $140 \mathrm{~cm}^{2}$, respectively. It is clear that very porous medium is generated in the portion where holes are generated in the later stage. 

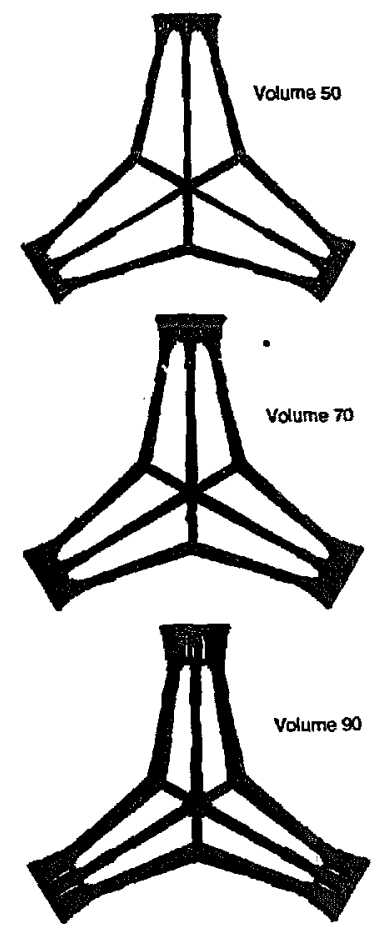
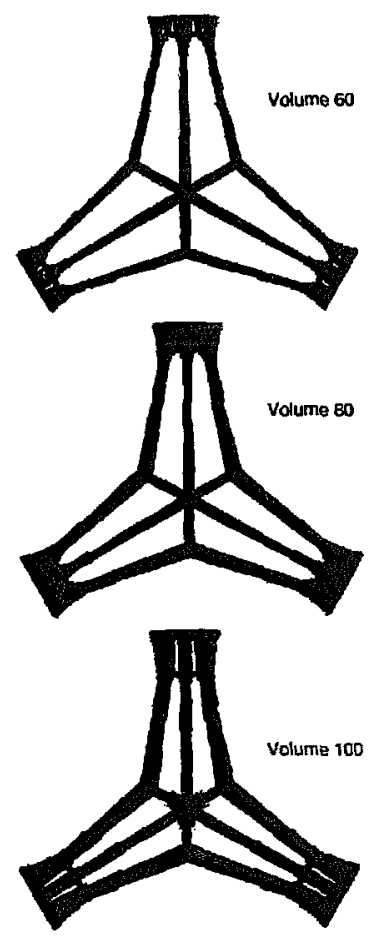
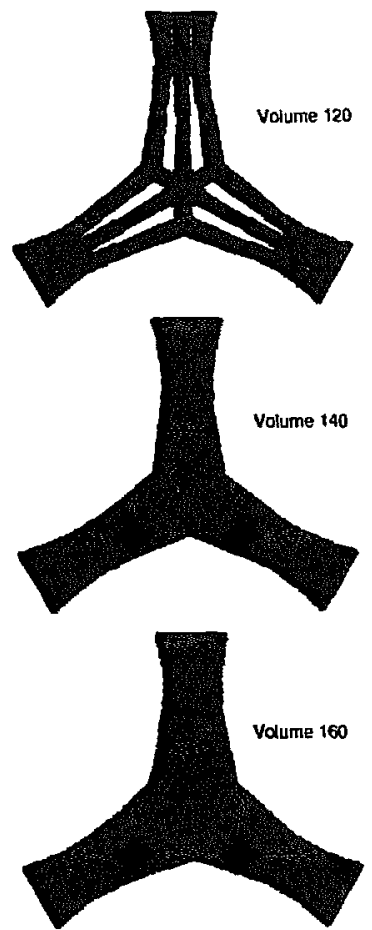
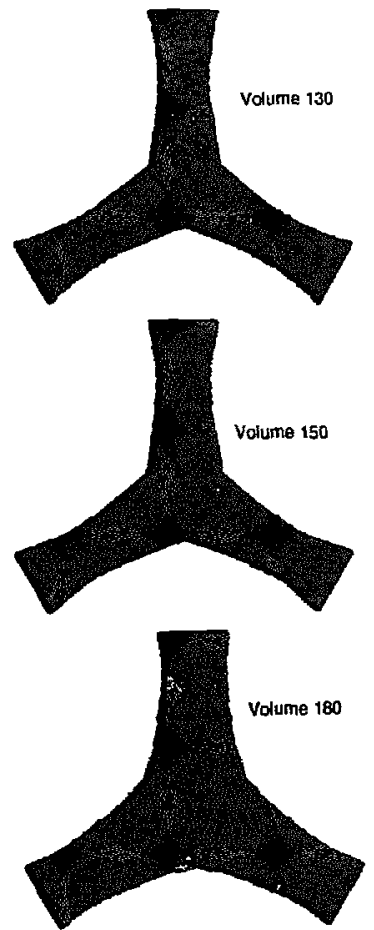

Fig. 28. Optimal configurations for various volumes of 'solid' material.

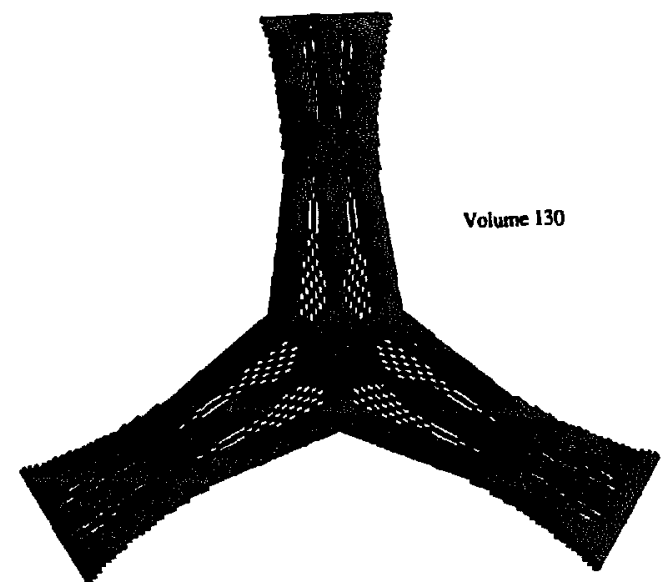

Fig. 29. Details of distribution of 'solid' material $\left(\Omega_{\mathrm{d}}=130 \mathrm{~cm}^{2}\right)$.

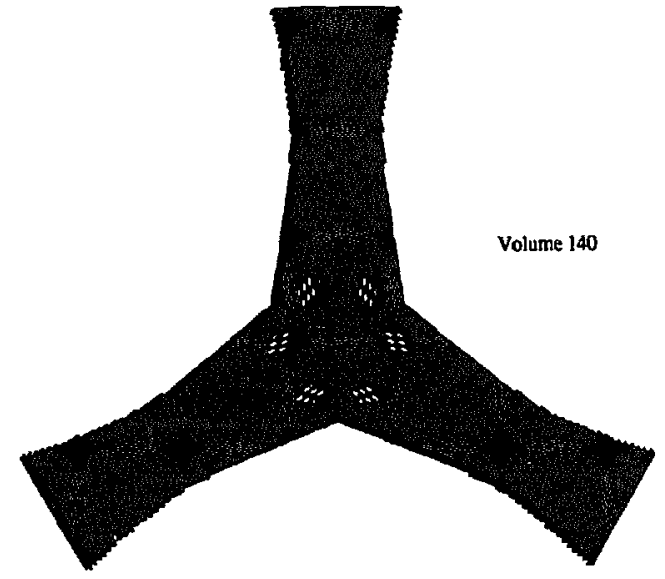

Fig. 30. Details of distribution of 'solid' material $\left(\Omega_{\mathrm{d}}=140 \mathrm{~cm}^{2}\right)$. 


\section{Multiple loading case}

As the last example shown in this work, we shall solve the optimal layout problem (7) for the case that several tractions are applied at the boundary at the same time. As shown above, tension and bending forces provide very different optimal configurations. Thus, it may be interesting in studying the case that several tractions whose nature is different are applied on the boundary. To do this, a rectangular domain is discretized by $64 \times 40$ rectangular finite elements, and is subject to the applied tractions as shown in Fig. 31. Tension, bending and shear forces are applied, while the bottom surface is assumed to be free here. The ratio of the shear-like traction and bending traction applied on the side surfaces is assumed to be one in the example.

Figures 32 and 33 show the optimal layout of the structure for $\Omega_{\mathrm{s}}=15 \mathrm{~cm}^{2}$ and $10 \mathrm{~cm}^{2}$. A solid medium is generated on the top surface while a very porous medium is formed in the sides. Overall configuration is an arch-type that can be seen in bridge or building structures. It is noted that the solid medium is generated in the portion where the bending stress is dominant, while the porous medium is formed in the region where the tensile and shear stresses are overwhelming.

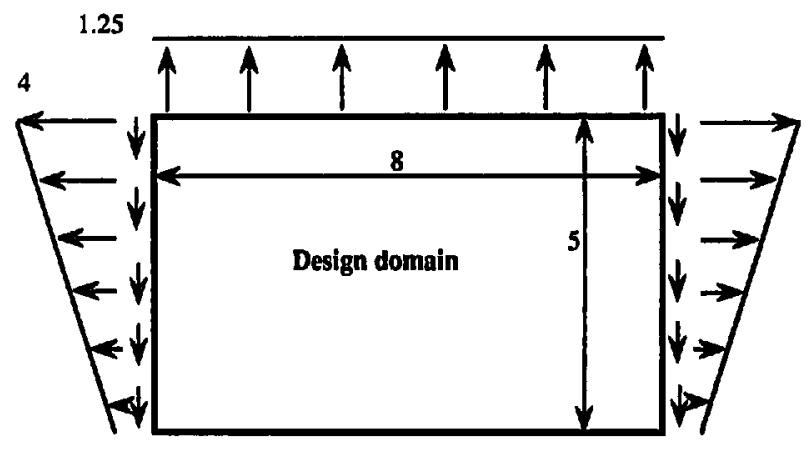

Fig. 31. Design domain and the applied loads ('shear'/ bending $=1 / 1$ ).

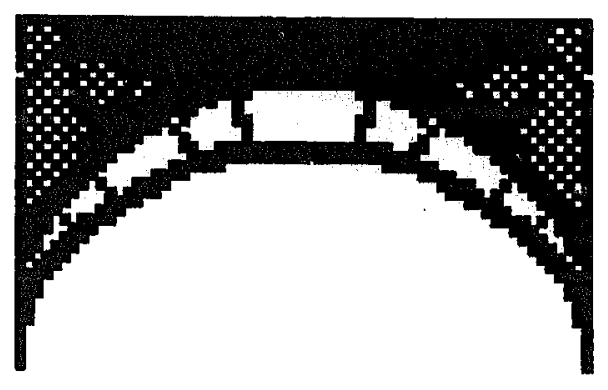

Fig. 32. Distribution of 'solid' material (solid/ void $=3 / 5$ ).

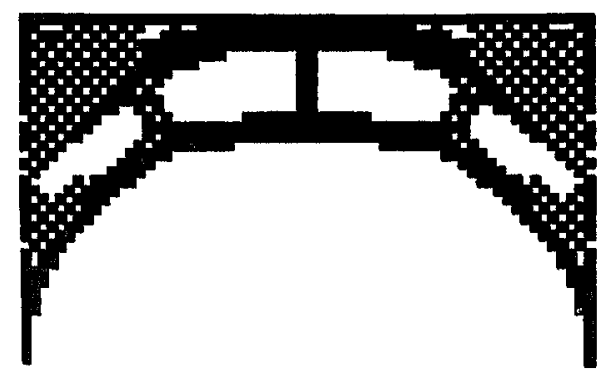

Fig. 33. Distribution of 'solid' material (solid/ void $=1 / 3$ ). 


\section{Acknowledgment}

The authors are supported partially by NASA Lewis Research Center, NAG 3-661, Office of Naval Research, ONR N-00014-88-K-0637 and Quint Co., Tokyo. They are grateful for these supports.

\section{References}

[1] L.A. Schmit, Structural design by systematic synthesis, Proc. 2nd ASCE Conf. Electronic Computation (ASCE, New York, 1960) 105-132.

[2] R.L. Fox, Constraint surface normals for structural synthesis techniques, AIAA J. 3 (8) (1965) 1517-1518.

[3] W. Prager and J.E. Taylor, Problems of optimal structural design, J. Appl. Mech. 35 (1968) 102-106.

[4] W. Prager, A note on discretized Michell structures, Comput. Methods Appl. Mech. Engrg. 3 (3) (1974) 349-355.

[5] A.G.M. Michell, The limits of economy of material in framed structures, Phil. Mag. 6 (1904) 589-597.

[6] K. Svanverg, Optimal geometry in truss design, in: A.J. Morris, ed., Foundations of Structural Optimization: A Unified Approach (Wiley, New York, 1982) Chapter 14, 513-544.

[7] G.I.N. Rozvany, Optimal Deisgn of Flexural Systems (Pergamon, Oxford, 1976).

[8] C. Fleury, Structural weight optimization by dual methods of convex programming, Internat. J. Numer. Methods Engrg. 14 (1979) 1761-1783.

[9] C. Fleury and G. Sander, Structural optimization by finite elements, LTAS Report SA-58, University of Liege, Belgium, 1978.

[10] J.S. Arora and E.J. Haug, methods of design sensitivity analysis is structural optimization, AIAA J. i9 (9) (1970) 970-974.

[11] D.E. Grierson, Computer automated design of building frameworks under various performance conditions, in: C.A. Mota Soares, ed., Computer Aided Optimal Design: Structural and Mechanical Systems (Springer, Berlin, 1987) 355-380.

[12] R.T. Haftka, Second order sensitivity derivatives in structural optimization, AIAA J. 20 (1982) 1765-1766.

[13] N. Olhoff, Optimal design with respect to structural eigenvalues, Proc. 15th Internat. Congress of Theoretical and Applied Mechanics, Toronto (1890) 133-149.

[14] K.T. Cheng and N. Olhoff, An investigation concerning optimal design of solid elastic plates, Internat. J. Solids and Structures 17 (1981) 305-323.

[15] O.C. Zienkiewicz and J.S. Campbell, Shape optimization and sequential linear programming, in: R.H. Gallagher and O.C. Zienkiewicz, eds., Optimal Structural Design (Wiley, New York, 1973) 109-126.

[16] R.T. Haftka and R.V. Gandhi, Structural shape optimization-A survey, Comput. Methods Appl. Mech. Engrg. 57 (1) (1986) 91-106.

[17] M.E. Botkin and J.A. Bennett, Shape optimization of three-dimensional folded-plate structures, AIAA J. 23 (11) (1985) 1804-1810.

[18] M.P. Bendsøe and N. Kikuchi, Generating optimal topologies in structural design using a homogenization method, Comput. Methods Appl. Mech. Engrg. 71 (2) (1988) 197-224.

[19] M.P. Bendsøe, Optimal shape design as a material distribution problem, Structural Optimization 1 (4) (1989) 193-202.

[20] G.I.N. Rozvany, Structural Design via Optimality Criteria (Kluwer Academic Publishers, Dordrecht, 1989).

[21] G.I.N. Rozvany, M. Zhou, M. Rotthaus, W. Gollub and F. Spengenmann, Continuum-type optimality criteria methods for large finite element system with a displacement constraint, Part I, Structural Optimization 1 (1) (1989) 47-72.

[22] P. Pedersen, On optimal orientation of orthotropic materials, Report \#375, The Danish Center for Applied Mathematics and Mechanics, The Technical University of Denmark, 1988, Lygby.

[23] L.V. Gibiansky and A.V. Chercaev, Microstructures of composites of external and exact estimates of provided energy density, Ioffe Science and Technology Institute, Leningrad, 1988. 
[24] G.I.N. Rozvany, T.G. Ong, N. Olhoff, M.P. Bendsøe, R. Sandler and W.T. Szeto, Least weight design of perforated elastic plates I and II, Internat. J. Solids and Structures 23 (4) (1987) 521-536, 537-550.

[25] P.G. Ciarlet, The Finite Element Method for Elliptic Problems (North-Holland, Amsterdam, 1978).

[26] J.T. Oden and J.N. Reddy, An Introduction to the Mathematical Theory of Finite Elements (Wiley/ Interscience, New York, 1976).

[27] W.S. Hemp, Chapter 4 Michell's structural continua, Optimal structures (Clarendon Press, Oxford, 1973) 70-101. 